\title{
Mesothelin/mucin 16 signaling in activated portal fibroblasts regulates cholestatic liver fibrosis
}

\author{
Yukinori Koyama, ${ }^{1,2,3}$ Ping Wang, ${ }^{1,2,4}$ Shuang Liang, ${ }^{1,2}$ Keiko Iwaisako, ${ }^{1,5}$ Xiao Liu, ${ }^{1,2}$ Jun Xu, ${ }^{1,2}$ Mingjun Zhang, ${ }^{1}$ Mengxi Sun, ${ }^{1,2}$ \\ Min Cong, ${ }^{1,2,4}$ Daniel Karin, ${ }^{1,2}$ Kojiro Taura, ${ }^{3}$ Chris Benner, ${ }^{1}$ Sven Heinz, ${ }^{1}$ Tapan Bera, ${ }^{6}$ David A. Brenner, ${ }^{1}$ and Tatiana Kisseleva ${ }^{2}$ \\ 'Department of Medicine and 'Department of Surgery, UCSD, La Jolla, California, USA. ${ }^{3}$ Department of Surgery, Graduate School of Medicine, Kyoto University, Kyoto, Japan. ${ }^{4}$ Beijing Friendship Hospital, \\ Capital Medical University, Beijing, China. ${ }^{5}$ Department of Target Therapy and Oncology, Graduate School of Medicine, Kyoto University, Kyoto, Japan. ${ }^{6}$ Laboratory of Molecular Biology, Center for Cancer \\ Research, National Cancer Institute, NIH, Bethesda, Maryland, USA.
}

\begin{abstract}
Cholestatic liver fibrosis is caused by obstruction of the biliary tract and is associated with early activation of portal fibroblasts (PFs) that express Thy-1, fibulin 2, and the recently identified marker mesothelin (MSLN). Here, we have demonstrated that activated PFs (aPFs) and myofibroblasts play a critical role in the pathogenesis of liver fibrosis induced by bile duct ligation (BDL). Conditional ablation of MSLN+ aPFs in BDL-injured mice attenuated liver fibrosis by approximately $50 \%$. Similar results were observed in MSLN-deficient mice ( $M s / n^{-/-}$mice) or mice deficient in the MSLN ligand mucin 16 (Muc16-/- mice). In vitro analysis revealed that MSLN regulates TCF- $\beta 1$-inducible activation of WT PFs by disrupting the formation of an inhibitory Thy-1-TCF $\beta$ RI complex. MSLN also facilitated the FGF-mediated proliferation of WT aPFs. Therapeutic administration of anti-MSLN-blocking Abs attenuated BDL-induced fibrosis in WT mice. Liver specimens from patients with cholestatic liver fibrosis had increased numbers of MSLN ${ }^{+}$aPFs/myofibroblasts, suggesting that MSLN may be a potential target for antifibrotic therapy.
\end{abstract}

\section{Introduction}

Fibrosis is an aberrant wound-healing process, in which chronic injury in any tissue activates myofibroblasts to produce a fibrous scar (1). Myofibroblasts are rare in normal tissues, and their origin depends on the tissue and type of injury. Many types of research suggest that liver-resident hepatic stellate cells (HSCs) and portal fibroblasts (PFs) are major sources of myofibroblasts in fibrotic liver (2). However, the composition of myofibroblasts varies significantly, depending on the etiology of hepatic fibrosis; hepatotoxic liver fibrosis (injury to hepatocytes, such as that caused by chronic viral hepatitis and nonalcoholic steatohepatitis) results from the activation of HSCs (3), while activated PFs (aPFs) are implicated in the pathogenesis of cholestatic fibrosis (obstruction to bile flow, such as that caused by primary and secondary biliary cirrhosis and primary sclerosing cholangitis) (4).

PFs are "periductular mesenchymal cells" that maintain the integrity of the portal tract $(5,6)$. In response to biliary obstruction, portal (myo)fibroblasts proliferate, upregulate expression of collagen type I, $\alpha$-smooth muscle actin ( $\alpha$-SMA), TGF- $\beta 1 / 2$, IL-6, and IL-13, and give rise to myofibroblasts (2, 7-14). Using a flow cytometry-based method of aPF purification (from bile duct ligation $[\mathrm{BDL}]-$ injured collagen- $\alpha 1[\mathrm{I}]-\mathrm{GFP}$ reporter mice, which express GFP under the control of the Col1a1 promoter) (4), we demonstrated that myofibroblasts derived from aPFs (referred to herein as aPFs/myofibroblasts) are a major source of collagen

Conflict of interest: The authors have declared that no conflict of interest exists. Submitted: June 9, 2016; Accepted: January 11, 2017.

Reference information: / Clin Invest. 2017;127(4):1254-1270.

https://doi.org/10.1172/JCl88845. type I-producing cells in early cholestatic liver injury, contributing more than $70 \%$ of the myofibroblasts by day 5 after BDL. The relative contribution of aPFs/myofibroblasts decreases with progressive injury, as HSCs become activated and contribute to the myofibroblast population (17 and 20 days after BDL). aPFs/ myofibroblasts express high levels of collagen type I, serve as a significant source of TGF- $\beta 2$, secrete IL-13 and IL-6 $(4,6)$, and facilitate the ductular reaction (cholestasis-induced proliferation of bile ducts) (7). aPFs are activated by taurocholic acid and IL-25, but unlike activated HSCs (aHSCs), are unresponsive to PDGF (4). aPFs can be distinguished from aHSCs by expression of Thy-1, fibulin 2, elastin, and the ecto-ATPase nucleoside triphosphate diphosphohydrolase 2 (NTPD2) and by their lack of aHSC markers (retinoid, desmin, glial fibrillary acidic protein [GFAP], p75NGFR) (15-18). Gene expression profiling of freshly isolated aPFs/myofibroblasts has identified several novel markers of aPFs, including mesothelin (MSLN) and mucin 16 (MUC16) (4).

MSLN (19), a membrane-anchored glycosylphosphatidyl inositol-linked (GPI-linked) 71-kDa membrane protein (MSLN precursor), is a mesothelial cell marker that is proteolitically cleaved to generate mature MSLN (40 kDa) and megakaryocyte-potentiating factor (MPF) (30 kDa) (20). Both molecules appear to be biologically active, although their exact function remains unknown. MSLN is expressed in several human malignancies, including mesotheliomas and ovarian cancer. Studies of patients with ovarian cancer have identified the cancer antigen CA125 as an MSLN ligand (20-23), which is widely used as a diagnostic marker (with the exception of pregnancy and liver cirrhosis, which are considered false-positives) (24). Since high expression of MSLN is linked to increased tumor proliferation and invasion, MSLN is a novel 
A

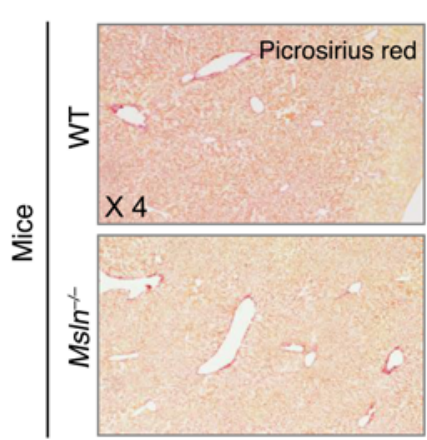

ALP

B

No injury

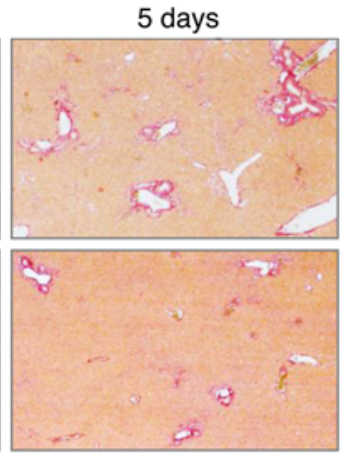

BDL

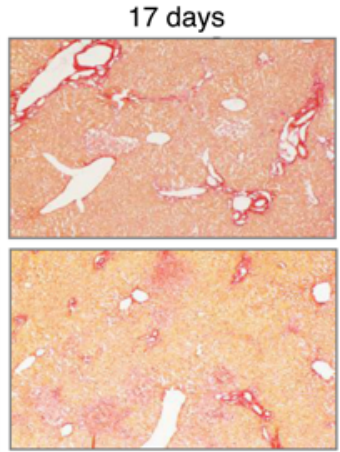

$\mathrm{CCl}_{4}$ 6 weeks

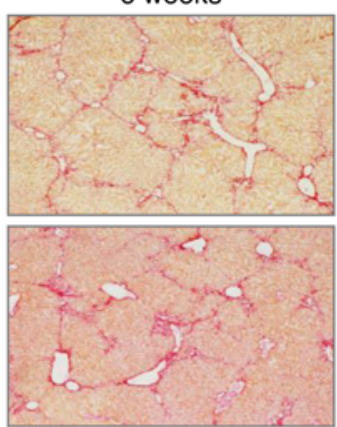

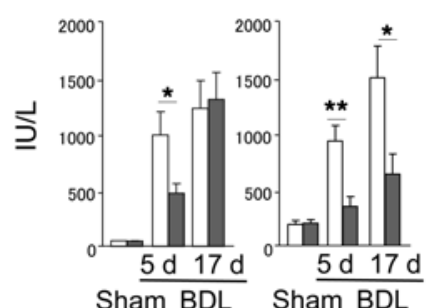
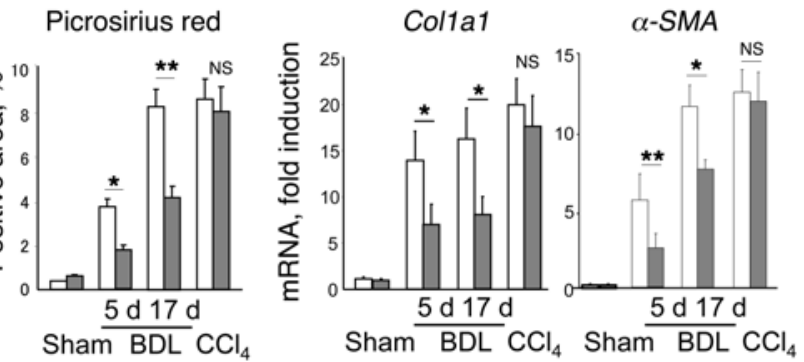

Col1a1

$\alpha-S M A$

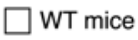

$M s / n^{-1}$ mice
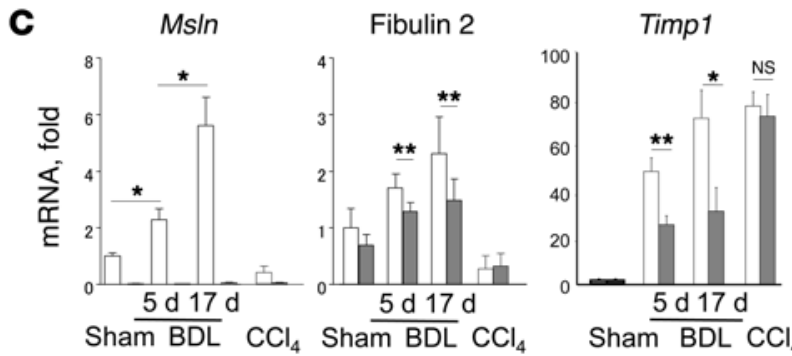

$F 4 / 80$

Tgfb1

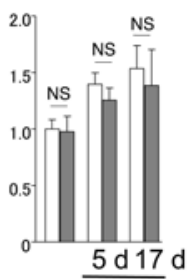

Sham BDL

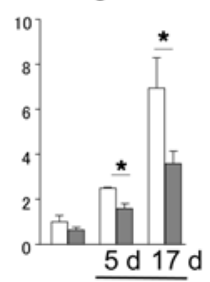

Sham BDL

Figure 1. Deletion of MsIn attenuates the development of cholestatic liver fibrosis in mice. (A-C) Eight-week-old male WT and Ms/n ${ }^{-/-}$mice (C57BL/6, $n=10$ /group, $n=3$ independent experiments) underwent sham or BDL operation (for 5 days or 17 days) or treatment with $C C_{4}(1.5 \mathrm{mo}, n=7 / \mathrm{group}$ ). Deletion of MsIn in mice attenuated BDL-induced (for 5 days) liver fibrosis by approximately $50 \%$. (A) Livers were stained with Picrosirius red, and (B) the positively stained area was quantified as a percentage using Image). (B) Liver function was estimated according to serum ALT and ALP levels (IU/I). (C) mRNA expression of fibrogenic (Col1a1, $\alpha$-SMA, Tgfb1), inflammatory (F4/80), and aPF-specific (Ms/n and fibulin 2) genes was determined by qPCR of total liver tissue. Data indicate the fold induction versus sham-operated WT mice. ${ }^{*} P<0.05$ and ${ }^{* *} P<0.01$, by 2-tailed Student's $t$ test.

target for anticancer therapy (25-29). In contrast to the embryonic mesothelium (30), MSLN is minimally expressed in adult humans and mice (20). MSLN-expressing cells reside in the mesothelial layer lining of parenchymal organs and serosal cavities (31) in a dormant state and do not proliferate until injured or stressed. Generation of $M s h^{C r-E R}$ mice enabled lineage tracing at early embryogenesis and linked expression of MSLN to fibroblast precursors (30). Although studies suggested that PFs and HSCs may originate from the hepatic mesothelium (32), the role of MSLN in the activation of PFs has not been evaluated. Furthermore, the molecular mechanisms underlying MSLN signaling in humans and mice are poorly understood. $\mathrm{Msln}^{-/-}$mice show no obvious abnormalities under physiological conditions (31). Similar results were obtained in MUC16-deficient mice (the murine analog of CA125) (33).

Here, we report that ablation of $\mathrm{MSLN}^{+}$aPFs/myofibroblasts, deletion of Msln in aPFs/myofibroblasts, or blocking of MSLN expression with anti-MSLN Abs attenuates BDL-induced cholestatic fibrosis in mice. Mechanistic studies identified what we believe to be a novel TGF- $\beta 1$ pathway, in which interaction of MSLN with a MUC16-Thy-1-TGFBRI complex regulates TGF- $\beta 1-$ mediated activation of aPFs/myofibroblasts. Analysis of patients' liver biopsies revealed that $\mathrm{MSLN}^{+} \mathrm{Thy}_{-1} \mathrm{1}^{+}$aPFs contribute to cholestatic fibrosis in patients with secondary biliary fibrosis and biliary atresia (BA), suggesting that MSLN may serve as an attractive target for antifibrotic therapy.

\section{Results}

$M$ sln-KO mice are resistant to BDL-induced cholestatic, but not hepatotoxic, liver fibrosis. To test the role of MSLN in the activation of aPFs/myofibroblasts, WT and $M s h^{-1-}$ mice were subjected to BDL and $\mathrm{CCl}_{4}$ injury (Figure 1, A-C). We observed no difference in liver fibrosis in $\mathrm{CCl}_{4}$-injured WT or Msln-- mice (at 1.5 months, Figure 1B). In concordance with this finding, the aPF markers MSLN and fibulin 2 were not upregulated in the livers of $\mathrm{CCl}_{4}$-injured mice 
A
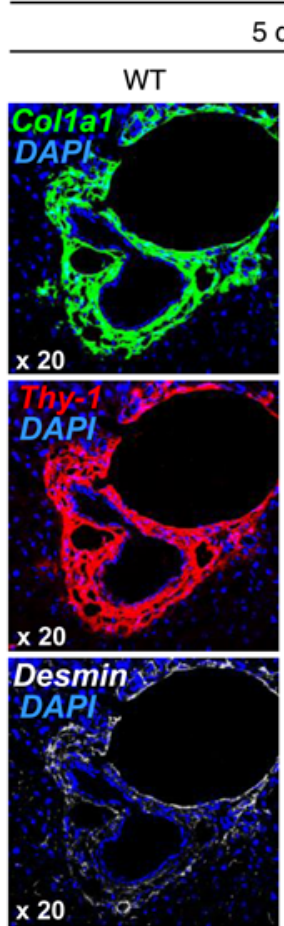

BDL

\section{5 days}
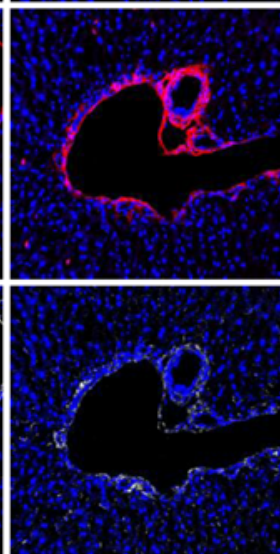

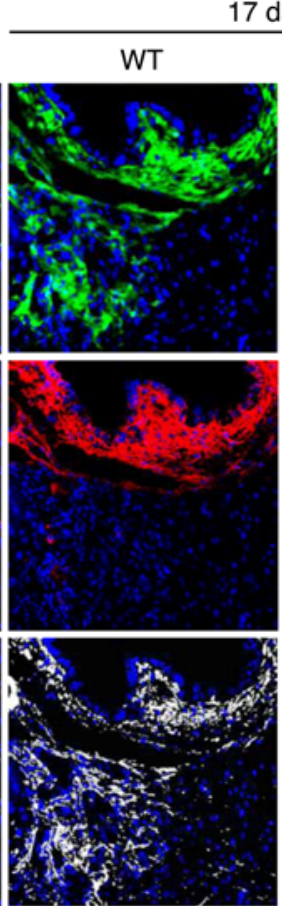

17 days
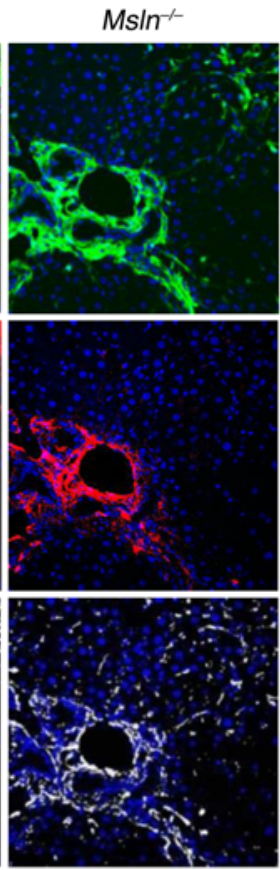

B

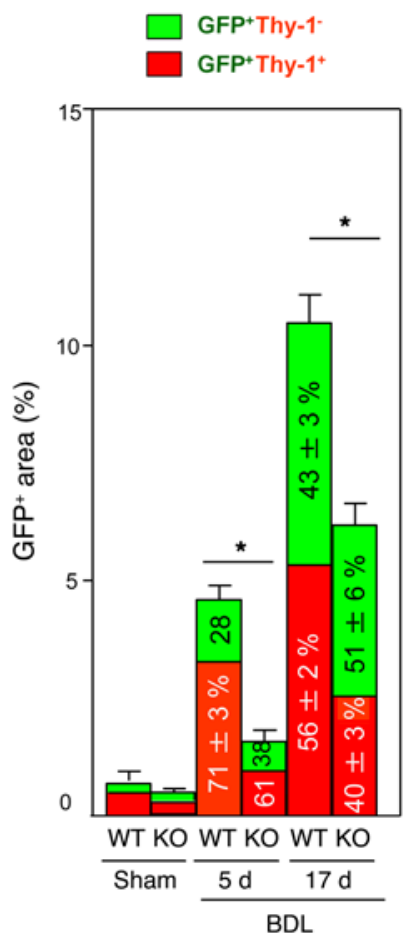

Figure 2. BDL-activated myofibroblast numbers are reduced in $\mathbf{M s} / \mathbf{n}^{-/-}$mice. (A and B) Eight-week-old male WT and $M s / n^{-/-}$mice ( $n=10 / g r o u p, n=3$ independent experiments) underwent sham or BDL operation (for 5 days or 17 days) or treatment with $\mathrm{CCl}_{4}(1.5 \mathrm{mo}, n=7 / \mathrm{group}$ ), and their livers were analyzed for expression of collagen- $\alpha 1$ (I)-GFP in myofibroblasts. (A) Livers were costained with DAPI, Thy-1, and desmin. (B) The area of overlapping $\mathrm{GFP}^{+}$Thy-1+ ${ }^{+}$staining was calculated (bars represent the percentage of positively stained area). The composition of GFP+ myofibroblasts (100\%) was determined as a percentage of aPFs (GFP+Thy-1+ red bars) and aHSC-derived (GFP+Thy-1- green bars). The appropriate controls for these images are provided in Supplemental Figure 1, B-D, refs. 64 and 65, and Supplemental Figure 2. ${ }^{*} P<0.05$, by 2-tailed Student's $t$ test.

(Figure 1C), confirming that aPFs/myofibroblasts do not activate in response to hepatotoxic liver injury.

In contrast, the development of BDL-induced cholestatic liver fibrosis was strongly attenuated in $\mathrm{Msln}^{-/-}$mice compared with that seen in WT mice (Figure 1, A-C). We observed significant downregulation of Picrosirius red staining (2-fold) and mRNA expression of the fibrogenic genes Col1a1 (2-fold), $\alpha$-SMA (1.7-fold), and Timp1 (1.7-fold) in the livers of $\mathrm{Msln}^{-/-}$mice 5 days after BDL, which correlated with low levels of serum alanine transaminase (ALT) and aspartate transaminase (ALP) (vs. levels in WT mice). We also observed inhibition of liver fibrosis in $\mathrm{Msln}{ }^{-/-}$mice on day 17 after BDL, as shown by decreased Picrosirius red staining ( $\downarrow 1.8$-fold) and downregulation of Col1a1 ( $\downarrow 1$.8-fold), $\alpha$-SMA ( $\downarrow 1.4$-fold), and Timp1 ( $\downarrow 1.7$-fold) mRNA expression, as well as reduced levels of serum ALP ( $\downarrow 2$-fold). Our data indicate that MSLN plays a critical role in the pathogenesis of cholestatic fibrosis, especially at the onset of BDL. Remarkably, hepatic expression levels of $\mathrm{F} 4 / 8 \mathrm{O}^{+}$ (Figure 1C), Tnfa, and Il1b mRNA (data not shown) were similar in BDL WT and $M s n^{-/-}$mice, indicating that inflammatory cells were not affected by MSLN deficiency.

Msln-KO mice are resistant to diethoxycarbonyl dihydrocollidineinduced cholestatic fibrosis. Similar results were obtained when WT and $\mathrm{Msln}^{-/-}$mice were subjected to another model of cholestatic liver injury induced by 3 weeks of diethoxycarbonyl dihydrocollidine (DDC) feeding. As expected, hepatic expression of Msln mRNA was strongly increased in DDC-fed WT mice (7-fold increase vs. control diet-fed WT mice) (Supplemental Figure 1A; supplemental material available online with this article; https://doi.org/10.1172/ JCI88845DS1). We observed significant downregulation of Picrosirius red staining ( $\downarrow 1$.7-fold decrease) and expression of the fibrogenic genes Col1a1 ( $\downarrow 2$-fold), $\alpha$-SMA ( $\downarrow 2$-fold), and Timp1 ( $\downarrow 1.7-$ fold) in the livers of $M s{ }^{-/-}$mice (Supplemental Figure 1A), which was associated with decreased expression of the aPF-specific gene fibulin 2. Our data indicate that MSLN critically contributes to the pathogenesis of cholestatic liver fibrosis of different etiologies.

The number of BDL-activated myofibroblasts was reduced in $\mathrm{Msln}^{-1-}$ mice. To further assess the role of MSLN in PF/myofibroblast activation, WT and $\mathrm{Msln}^{--}$mice were crossed with reporter collagen $\alpha 1$ (I)GFP mice (in which all activated myofibroblasts upregulate collagen $\alpha 1$ (I)-GFP expression) and subjected to sham or BDL treatment (5 and 17 days of BDL), Figure 2, A and B, and Supplemental Figure 1, B-D). Livers were costained with Thy- 1 and desmin to visualize aPF- and aHSC-derived myofibroblasts, respectively (Figure 2A), and the area of Thy $-1^{+} \mathrm{GFP}^{+}$and Thy-1-GFP ${ }^{+}$costaining was calculated (Figure $2 \mathrm{~B}$ ).

Although expression of collagen $\alpha 1$ (I)-GFP was increased in both WT and $\mathrm{Msln}^{-/}$mice following BDL (5 days of BDL vs. sham-operated mice), the number of activated $\mathrm{GFP}^{+}$myofibroblasts was reduced by nearly 3-fold in BDL-injured $\mathrm{Msln} \mathrm{n}^{-/}$ mice $(1.6 \% \pm 0.2 \%$ vs. $4.8 \% \pm 0.2 \%$ in BDL-treated WT mice). Meanwhile, the composition of $\mathrm{GFP}^{+}$myofibroblasts (considered $100 \%$ ) was not significantly changed between WT and $M s n^{-1-}$ mice after 5 days of BDL. Thus, WT GFP myofibroblasts 

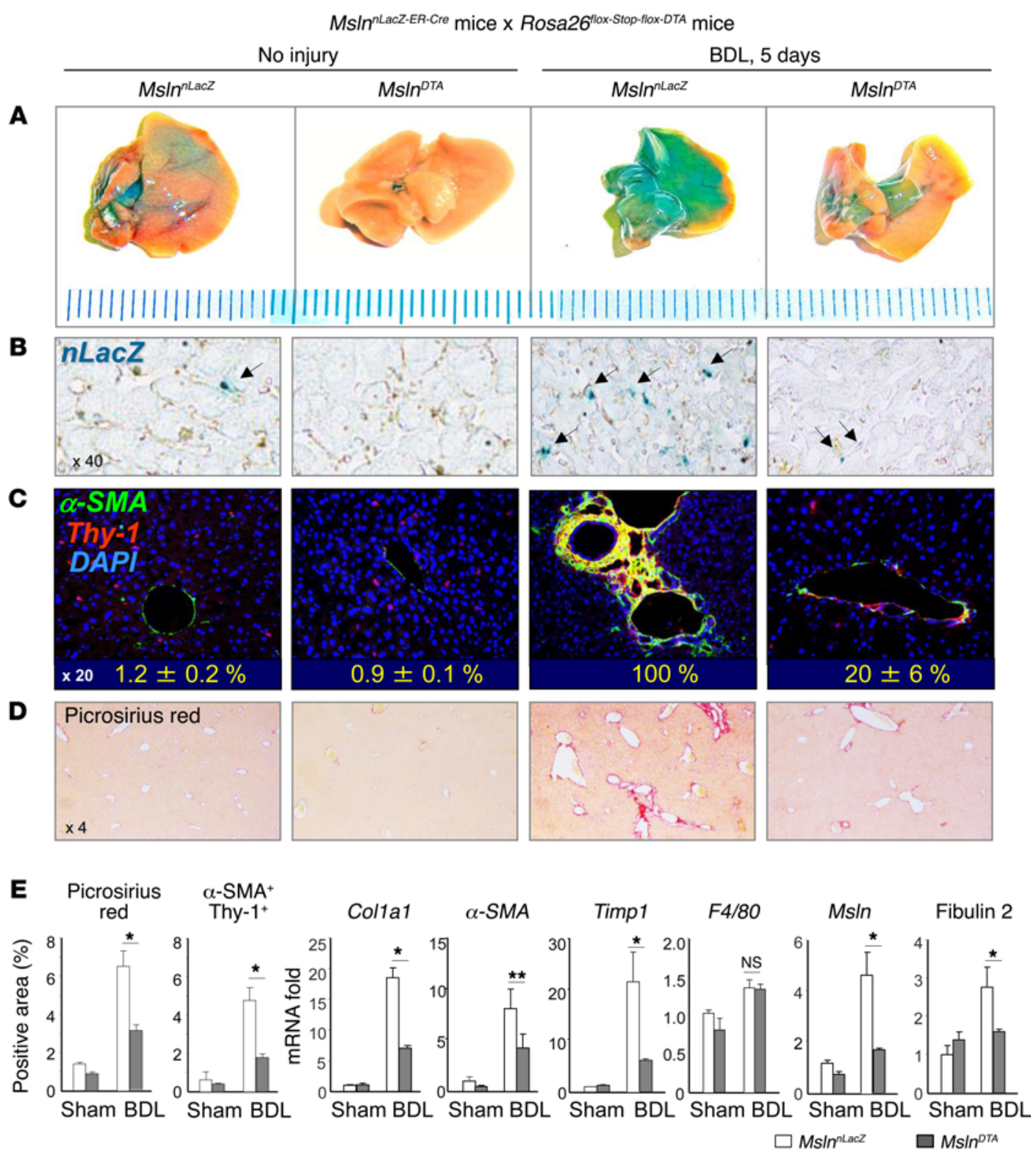

Figure 3. Ablation of MsIn attenuates development of cholestatic liver fibrosis in mice. (A) Tamoxifen administration ( $12 \times 5 \mathrm{mg} / \mathrm{mouse}$ by oral gavage) irreversibly labeled all MSLN ${ }^{+}$aPFs by nuclear LacZ ( $\left.n L a c Z\right)$ expression in WT Ms $/ n^{\text {LLacZ }}$ mice $(n=14)$ and caused ablation of aPFs in Ms/ $n^{\text {DTA }}$ littermates (C57BL/6, 8-week-old male mice, $n=17$ from 3 independent experiments). Livers were stained for LacZ (micrographs show gross liver tissue) and (B) analyzed for LacZ expression (original magnification, $\times 40$ ). (C) Liver sections were immunostained with DAPI for Thy-1 and $\alpha$-SMA expression. The number of aPFs in sham-operated $M s / n^{\text {nLacZ }}$ and $M s / n^{D T A}$ mice and the efficiency of aPF/myofibroblast ablation in BDL-operated Ms/n ${ }^{\text {DTA }}$ mice were calculated in comparison with the number of Thy $-1^{+} \alpha-S M A^{+} D A P I^{+}$cells in livers of BDL-Ms/n $n^{\text {nLacz }}$ mice (considered as $100 \%$ ). Representative micrographs are shown (original magnification, $\times 20$ ). (D) Livers were stained with Picrosirius red (original magnification, $\times 4$ ). (E) Quantification of Picrosirius red ${ }^{+}$and Thy-1 $1^{+} \alpha-S M A^{+}$areas is shown as a percentage. Expression of fibrogenic, aPF-specific, and inflammatory gene mRNA was analyzed by qPCR and is shown as a fold induction. ${ }^{*} P<0.05$ and ${ }^{* *} P<0.01$, by 2 -tailed Student's $t$ test. (See also Supplemental Figure 3.)

were composed of $71 \% \pm 3 \%$ aPFs and $28 \% \pm 2 \%$ aHSCs, while MSLN-deficient $\mathrm{GFP}^{+}$myofibroblasts were composed of $61 \% \pm$ $2 \%$ aPFs and $38 \% \pm 4 \%$ aHSCs.

Expression of MSLN has been reported in both aPFs/myofibroblasts and hepatic mesothelial cells, identified by expression of the GPM6A marker (34). Costaining with anti-glycoprotein M6A (anti-GPM6A) Ab revealed that mesothelial cells contribute to a small number of collagen $\alpha 1(\mathrm{I})-\mathrm{GFP}-$ positive $\left(\mathrm{Col}^{+}\right)$ myofibroblasts residing in the liver capsule of the WT mice sub- jected to 5 days of BDL injury (Supplemental Figure 2A), while the majority of $\mathrm{Thy}^{-1} \mathrm{CO}^{+}$myofibroblasts were located in the portal areas and lacked GPM6A expression (Supplemental Figure $2 \mathrm{~B}$ ). Since no other cell types in the liver (including HSCs, cholangiocytes, endothelial cells, hepatocytes, and Kupffer cells) (Supplemental Figure 2, C and D) were reported to coexpress collagen $\alpha 1(\mathrm{I})$ and MSLN (and Thy-1), the loss of activated myofibroblasts in BDL-injured $\mathrm{Msln}^{-/-}$mice was attributed to the MSLN deficiency in aPFs/myofibroblasts. 
A

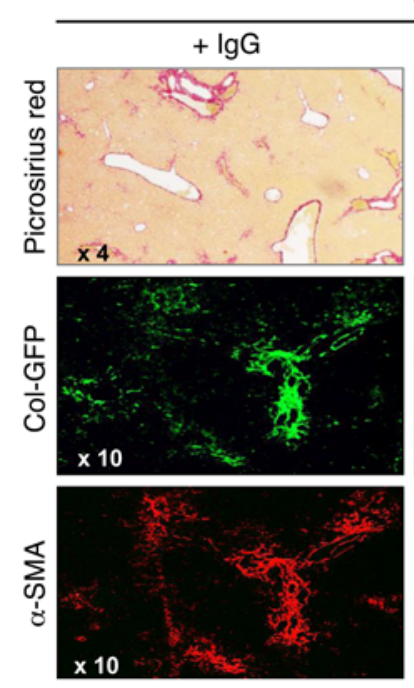

Col-GFP mice, BDL (5 days)
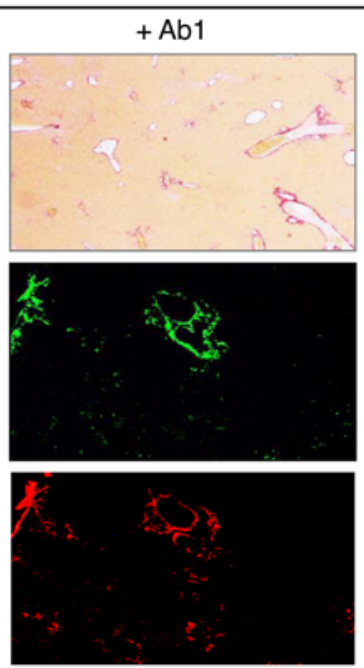
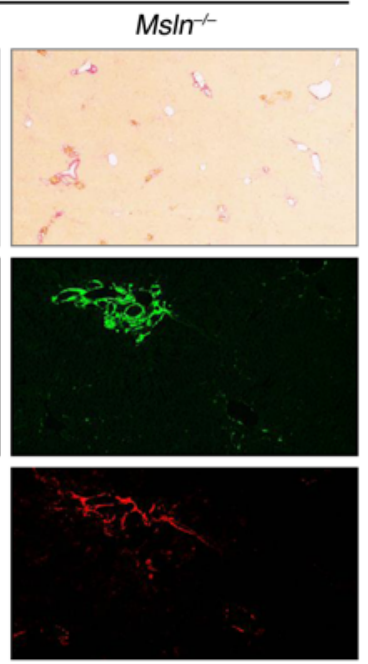

B

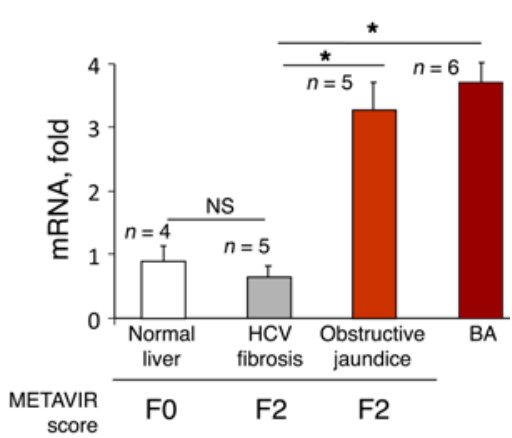

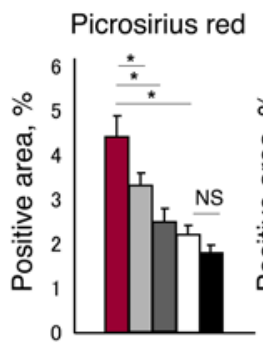
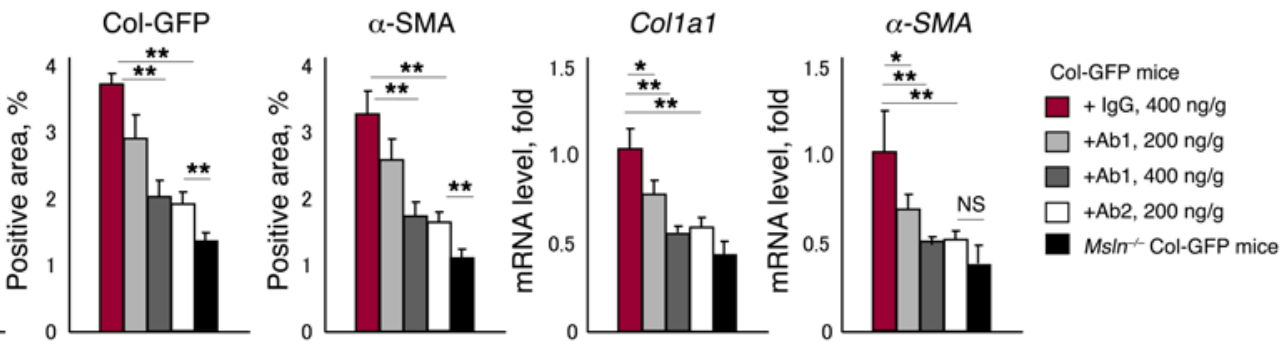

Figure 4. Therapeutic administration of anti-MSLN Abs reduces cholestatic fibrosis development in mice. (A) Liver fibrosis was reduced in BDL-injured 8-week-old male collagen $\alpha 1$ (I)-GFP (Col-GFP) mice (C57BL/6, $n=10 /$ group, $n=2$ independent experiments) that were treated with anti-MSLN-blocking Abs (2 i.p. injections; Ab1: 200 or 400 ng/g BW; Ab2: 400 ng/g BW, administered 24 and 48 hours after BDL) compared with lgG-treated (400 ng/g BW) and Ms/n ${ }^{-/}$ mice $(n=3)$. Micrographs show Picrosirius red, $\alpha$-SMA, and Col-GFP staining (original magnification, $x 4$ and $\times 10)$. The positively stained area was calculated as a percentage. Col1a1 and $\alpha$-SMA mRNA expression levels are shown as fold downregulation. ${ }^{*} P<0.05$ and ${ }^{* *} P<0.01$, by ANOVA. (B) Expression of human MSLN mRNA was analyzed by qPCR in liver specimens from patients with BA $(n=6)$; secondary biliary fibrosis (METAVIR score of F2, $n=5$ ); and HCV fibrosis (METAVIR score of F2, $n=5$ ) versus control livers $(n=4) .{ }^{*} P<0.05$, by ANOVA. (See also Supplemental Figure 4.)

Ablation of $M S L N^{+}$aPFs attenuates BDL-induced liver fibrosis. MSLN was minimally expressed in adult mice but was strongly upregulated in response to BDL (Figure 1C). To gain insight into the role of $\mathrm{MSLN}^{+} \mathrm{aPFs} /$ myofibroblasts in the pathogenesis of cholestatic liver fibrosis, we generated mice devoid of $\mathrm{MSLN}^{+}$cells. We assumed that, if MSLN ${ }^{+}$aPFs significantly contribute to cholestatic liver fibrosis, then ablation of $\mathrm{MSLN}^{+}$aPFs would attenuate the development of cholestatic liver fibrosis. To examine this, $M s \ln ^{\text {DTA }}$ mice ( $M s l^{n L L a c Z}$ mice, in which the cassette harboring the reporter nuclear [n]LacZ and tamoxifen-inducible ER-Cre was knocked into the $M s \ln$ gene) were crossed with Rosa26 fox-Stop-flox-DTA mice (Figure 3A). The resulting MSLN-ablated $M s{ }^{D T A}$ mice and WT $M s l h^{n L a c z}$ littermates were treated with tamoxifen and then subjected to 5 days of BDL injury. Upon administration of tamoxifen to $M s l \eta^{n L a c Z}$ mice, $\mathrm{MSLN}^{+}$aPFs/myofibroblasts had increased $\mathrm{nLacZ}^{+}$ expression and were scattered throughout the liver parenchyma of $M s l h^{n L a c Z}$ mice (Figure 3B and Supplemental Figure 3A). In turn, the disappearance of hepatic nLacZ ${ }^{+}$expression in $M s l n^{\text {DTA }}$ mice was indicative of successful ablation of $\mathrm{MSLN}^{+} \mathrm{aPFs} /$ myofibroblasts and correlated with marked downregulation of the aPF-specific markers MSLN and fibulin 2 in the livers of $M s \ln ^{\text {DTA }}$ mice (Figure $3 \mathrm{E}$ ). DAPI costaining for overlapping expression of $\alpha$-SMA and Thy- 1 confirmed that deletion of more than $80 \%$ of the Thy- $1^{+} \alpha-\mathrm{SMA}^{+}$ myofibroblasts was achieved in BDL-Msln ${ }^{D T A}$ mice (vs. BDL-treated $M s l n^{n L a c Z}$ mice) (Figure 3C and Supplemental Figure 3B).

We compared the development of cholestatic fibrosis in $M s n^{\text {DTA }}$ and WT Msln ${ }^{\text {nLacz }}$ mice 5 days after BDL surgery and found that ablation of $\mathrm{MSLN}^{+} \mathrm{aPFs} /$ myofibroblasts attenuated liver fibrosis by approximately $50 \%$, as demonstrated by the reduced area of Picrosirius red staining and reduced expression of Col1a1, $\alpha$-SMA, and Timp1 mRNA (Figure 3, D and E). We concluded that MSLN-expressing aPFs/myofibroblasts contribute to cholestatic liver fibrosis and that therapeutic targeting of $\mathrm{MSLN}^{+} \mathrm{aPFs} /$ myofibroblasts might attenuate BDL-induced fibrosis in mice.

Delayed administration of anti-MSLN Abs inhibits cholestatic fibrosis in mice. To test whether anti-MSLN-blocking Abs can avert the development of cholestatic fibrosis, BDL-operated collagen $\alpha 1(\mathrm{I})$-GFP mice were treated with 2 consecutive doses of antiMSLN Abs or isotype IgG (24 and 48 hours after BDL) (Figure 4A) and sacrificed 5 days after BDL surgery. Delayed administration of Ab no. 1 (Ab1) (200 ng/g or $400 \mathrm{ng} / \mathrm{g} \mathrm{BW;} \mathrm{Abbiotec;} \mathrm{250519)} \mathrm{to}$ BDL-treated collagen $\alpha 1$ (I)-GFP mice inhibited fibrosis and activation of $\mathrm{GFP}^{+}$myofibroblasts in a dose-dependent manner. Similar results were achieved using delayed administration of Ab no. 
A

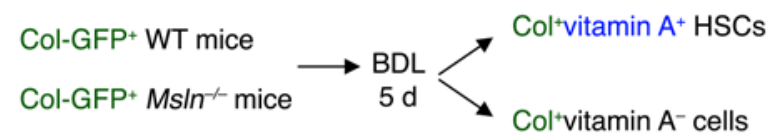

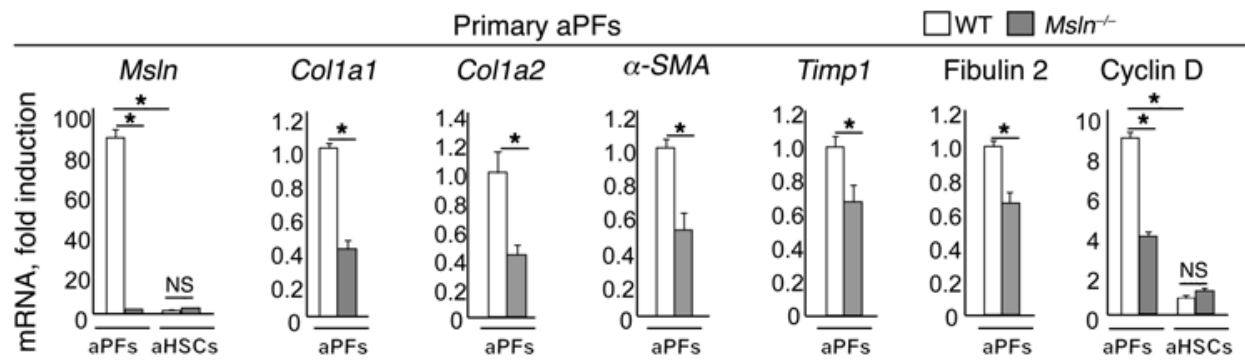

B Primary aPFs
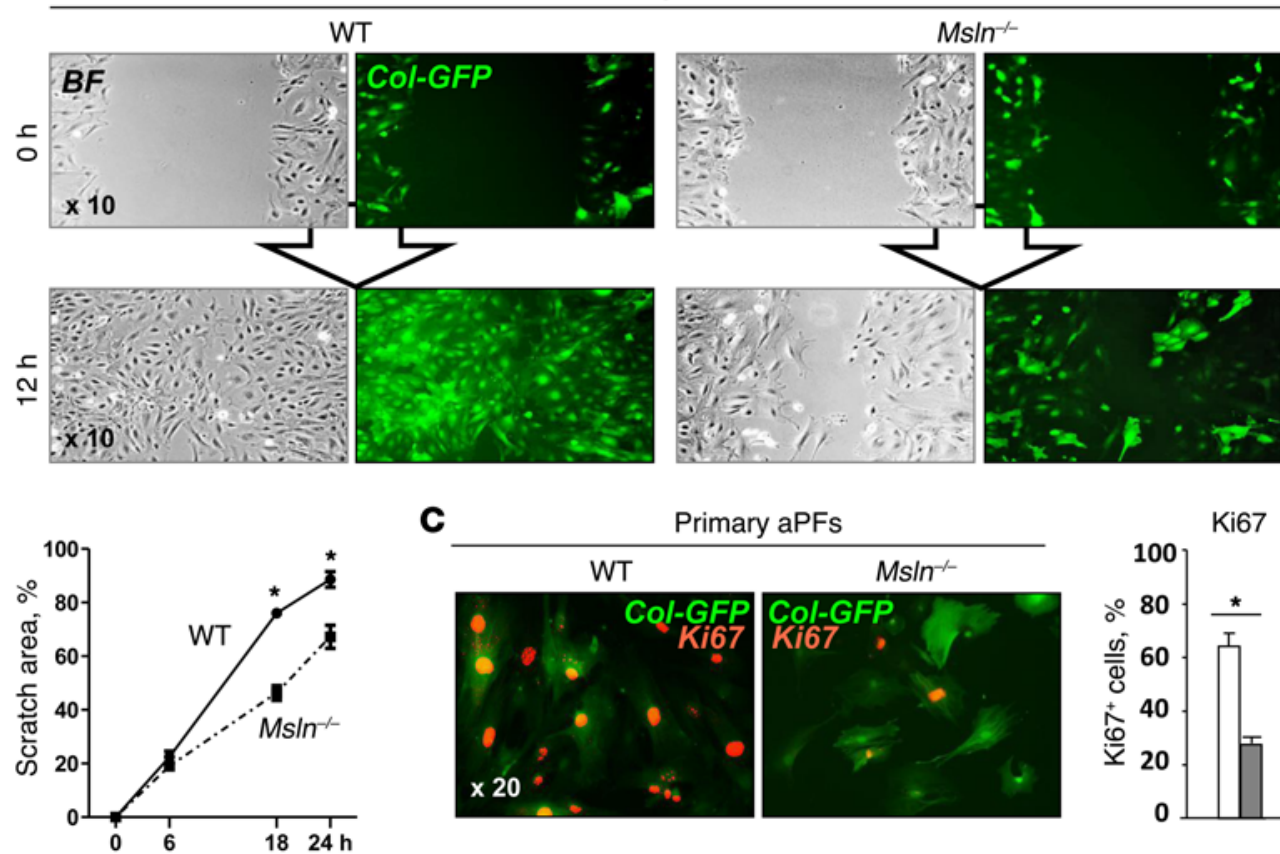

c

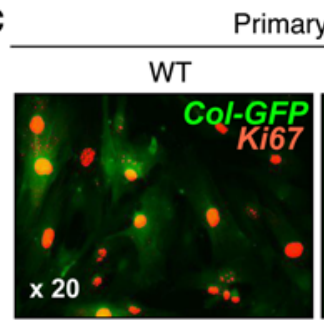

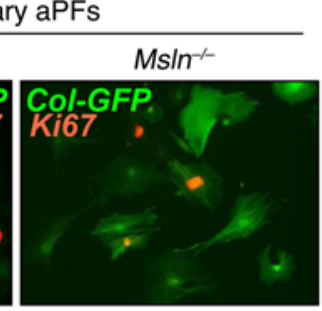

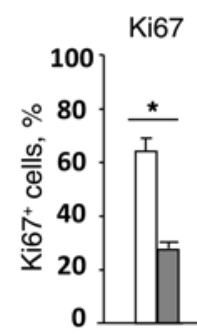

Figure 5. Primary MsIn ${ }^{-/-}$aPFs have a defect in activation and proliferation. (A) Primary GFP ${ }^{+}$vitamin $\mathrm{A}^{-}$aPFs were sort purified from the livers of BDLinjured ( 5 days) WT and $M s / n^{-1-}$ mice ( $n=3 /$ isolation, 6 independent experiments), and mRNA expression of lineage-specific genes (Supplemental Figure 5) and activation markers was analyzed by qPCR. (B) Primary WT and Ms/n ${ }^{-1-}$ aPFs (10 $/$ well) were assessed in a 12 -hour scratch assay. Shown are representative bright-field (BF) and fluorescence (Col-GFP) micrographs (original magnification, $\times 10$ ). The scratch area was calculated as a percentage (average of $>3$ independent experiments). (C) Ki67 immunostaining of primary WT and Ms/n ${ }^{-1-}$ aPFs within the scratch area. Representative images (original magnification, $\times 20$ ) are shown. Graph shows the percentage of Ki67+ aPFs versus total aPFs $(100 \%)$ for an average of 3 experiments. ${ }^{*} P<0.05$, by 2 -tailed Student's $t$ test or ANOVA for comparisons between 2 groups or more than 2 groups, respectively.

2 (Ab2) (400 ng/g BW; MBL; D233-3), suggesting that targeting MSLN may be beneficial for patients with cholestatic liver fibrosis. In support of this, several classes of anti-MSLN Abs $(26,35)$ have been generated and are currently being used in clinical trials.

Expression of human MSLN is upregulated in patients with cholestatic liver fibrosis. To gain insight into the role of aPFs in human liver diseases, the myofibroblast composition was assessed in resected livers from patients with cholestatic liver fibrosis (BA, $n=6$; secondary biliary cirrhosis, $n=12$; and hepatotoxic $\mathrm{HCV}, n=5$ ) using quantitative PCR (qPCR) using immunohistochemical analyses (Figure 4B and Supplemental Figure 4). Expression of MSLN mRNA was upregulated by nearly 3 -fold in patients with cholestatic fibrosis (but not in those with nonfibrotic or HCV-fibrotic livers). Immunostaining for MSLN and Thy- $1^{+}$expression was detected only in fibrotic lesions of patients with cholestatic fibrosis and overlapped with $\alpha-\mathrm{SMA}^{+}$(but not desmin ${ }^{+}$) staining, suggesting that MSLN can serve as a marker of human aPFs (Supplemental Figure 4) and mouse aPFs (4).

Primary Msln ${ }^{-/}$aPFs/myofibroblasts have a defect in activation and proliferation. Our findings suggest that genetic and pharmacological ablation of $\mathrm{MSLN}^{+} \mathrm{aPFs} /$ myofibroblasts or genetic deletion of $M s \ln$ in mice attenuates the development of cholestatic liver fibrosis (Figures 1 and 4). To investigate the role of MSLN in aPF/myofibroblast activation, primary aPFs were sort purified from BDL-injured (for 5 days) livers of collagen $\alpha 1$ (I)-GFP WT and collagen $\alpha 1$ (I)-GFP $\mathrm{Msln}^{-/-}$mice and phenotyped (Supplemental Figure 5). In comparison with WT aPFs, we found that expression of fibrogenic genes was 
A

Immortalized aPFs $\square$ WT $\square M^{\prime} \ln ^{-1}$

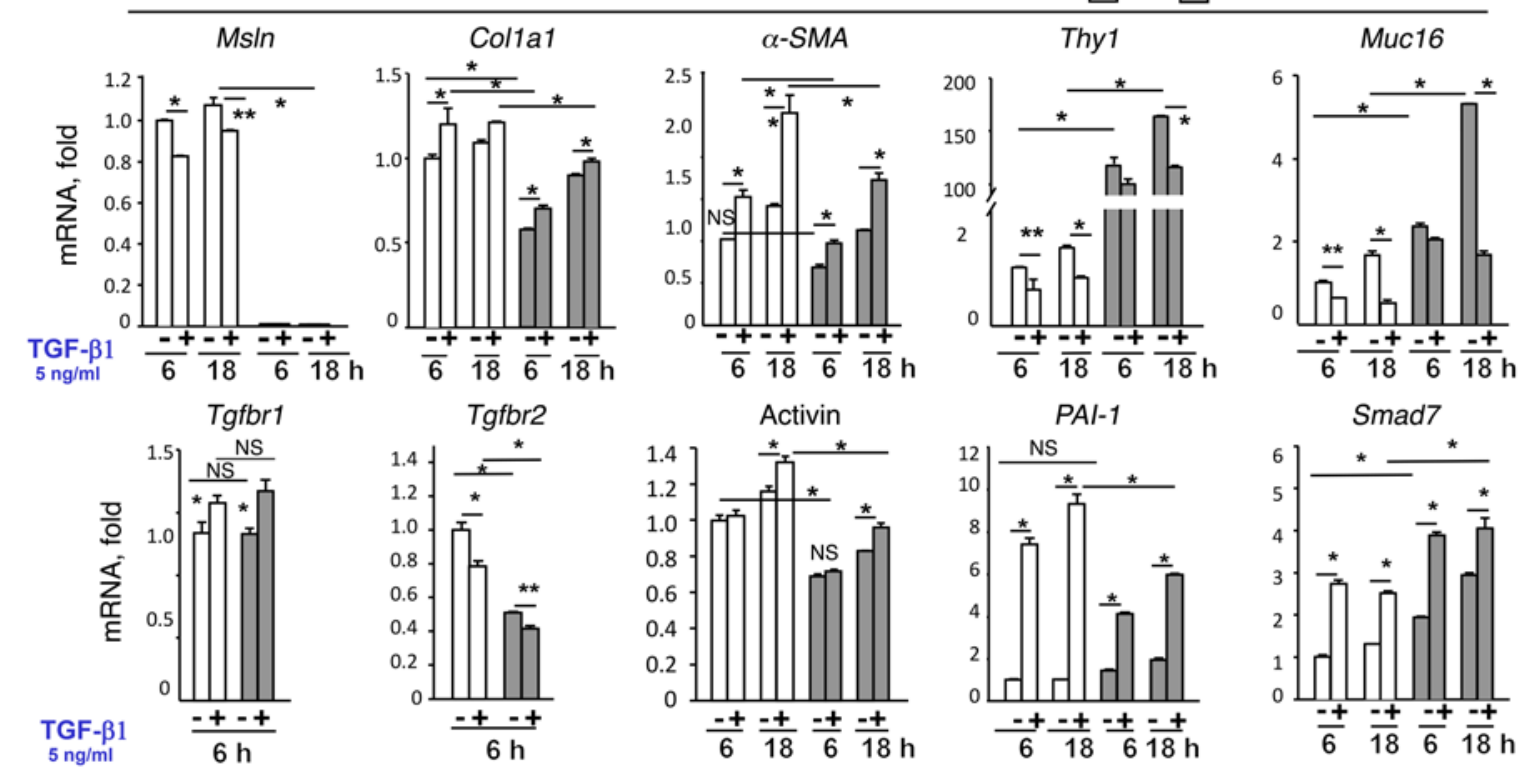

B

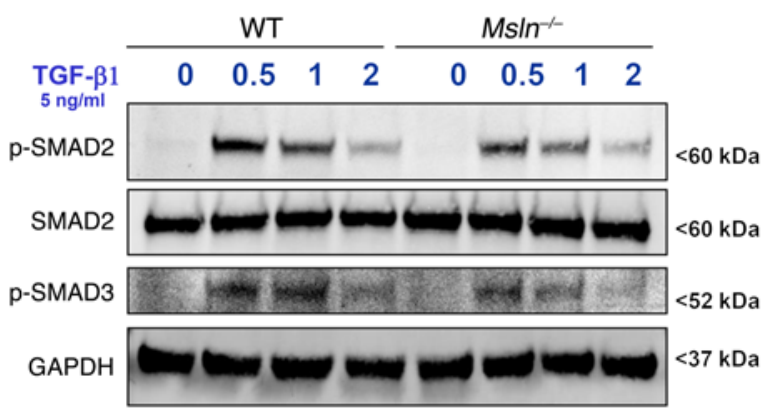

D

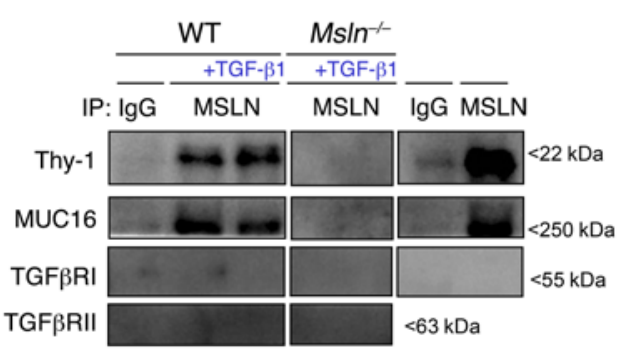

C

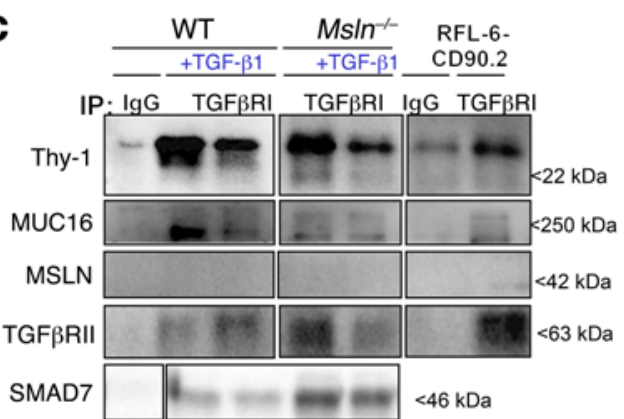

E

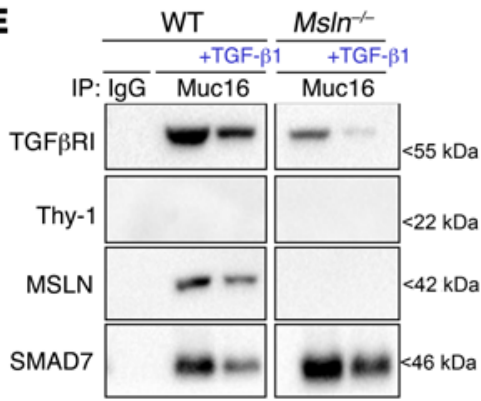

$\mathbf{F}$
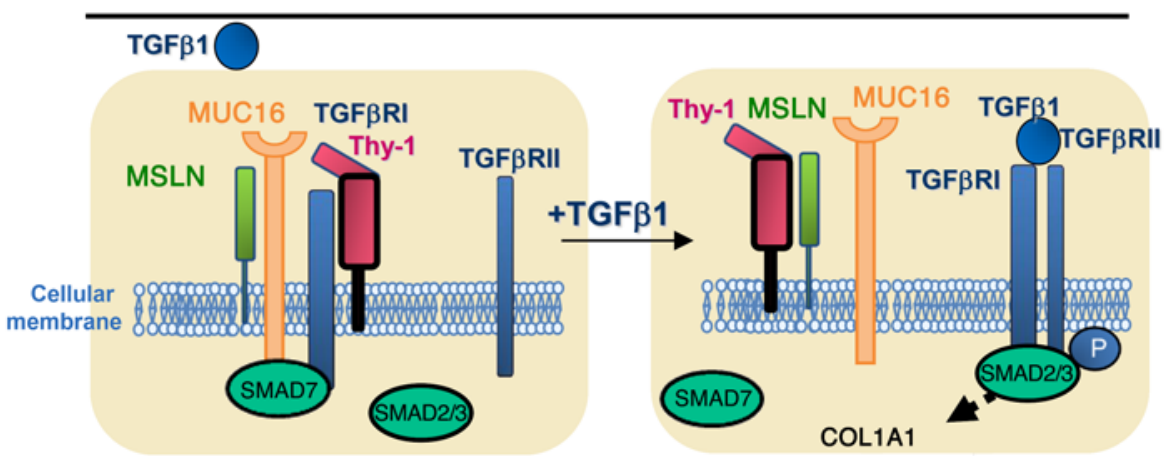

$M s / n^{-/}$aPFs

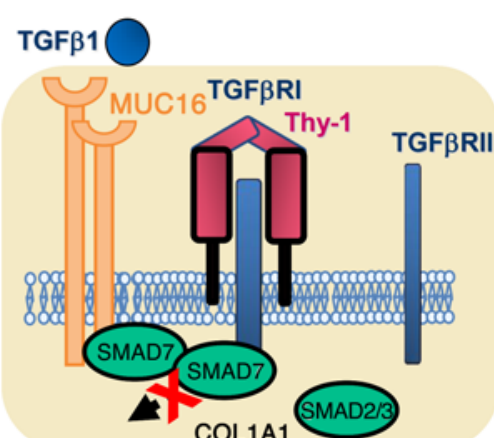


Figure 6. Ms/n ${ }^{-/-}$aPFs exhibit defective TGF- $\beta 1$ signaling. (A) Responses to TCF- $\beta 1$ were compared in immortalized WT and $M s / n^{--}$aPFs using qPCR. $P$ values were determined by ANOVA, ${ }^{* *} P<0.01$ and ${ }^{*} P<0.05$. (B) TCF- $\beta 1$-induced phosphorylation of SMAD2/3 was compared in WT and $M s / n^{-1-}$ aPFs using Western blotting (representative blots are shown). (C-E) IPs of cell lysates from WT or Ms/n ${ }^{-1-}$ aPFs, with or without TCF- $\beta 1$, and of lysates of RFL-6-CD90.2 (lung fibroblast cell line) and LMP (pancreatic cancer cell line), which were used as controls for the expression of Thy- 1 and MSLN, respectively, with (C) anti-TGF $\beta R I$ Ab, (D) anti-MSLN Ab, and (E) anti-MUC16 Ab. Immunoblots are representative of more than 3 independent IPs (see also Supplemental Figure 6). (F) Proposed model of MSLN regulation of TGF- $\beta 1$ signaling. In WT aPFs, Thy-1, and MUC16 interact with TCF $\beta R I$ under steady-state conditions, and MUC16 and Thy-1 bind to MSLN (but not to each other). In TGF- $\beta 1$-stimulated WT aPFs, both Thy- 1 and MUC16 dissociate from the TCF $\beta R$ I complex, and MSLN-Thy-1 interaction is increased, while MSLN-MUC16 interaction is decreased, suggesting that binding of MSLN to Thy-1 enables TGF- $\beta 1$ signaling. Deletion of MsIn results in overexpression of Thy-1, MUC16, and SMAD7 in Ms/n ${ }^{-1-}$ aPFs, increased formation of the inhibitory Thy-1-TCF $\beta R I$ complex, and retention of SMAD7 at the C-terminus of MUC16 TCF $\beta R$ I receptors.

downregulated in $M s{ }^{-/-}$aPFs (fold decrease of 2.5 for Col1a1, 2.5 for Col1a2, 2 for $\alpha$-SMA, and 1.5 for Timp1) (Figure 5A). Expression of cyclin D was also decreased (2.5-fold), suggesting that $\mathrm{Msln}^{-1}$ aPFs might have a proliferative defect. Furthermore, we performed a scratch assay to examine the proliferation and migration of WT and $\mathrm{Msln}^{-/}$aPFs (Figure 5B) and found that, unlike WT PFs, Msln ${ }^{-1}$ aPFs failed to populate the scratch area within 12 hours and showed a defect in expression of the proliferation marker Ki67 (Figure 5C). Our data suggest that MSLN-deficient aPFs/myofibroblasts exhibit defective activation and proliferation.

Immortalized $\mathrm{Msln}^{-/-}$aPFs exhibit defective TGF- $\beta 1$ signaling. Next, we investigated the role of MSLN in the activation of aPFs/ myofibroblasts using immortalized WT and $M s n^{-/-}$aPFs (generated by introducing SV40 large T antigen). Like primary $\mathrm{Msln}^{-1}$ aPFs, immortalized $M s{ }^{-/-}$aPFs had defective TGF- $\beta 1$ signaling. Induction of the TGF- $\beta 1$ target genes Col1a1, $\alpha-S M A, P A I-1$, and activin (Figure 6A) was reduced in TGF- 31 -stimulated $\mathrm{Msln}^{-1}$ aPFs and was associated with impaired phosphorylation of the SMAD2/3 complex (Figure 6B) as compared with WT aPFs. In addition, $M s l^{-1-}$ aPFs had upregulated expression of Thy-1 (150fold), Muc16 (2.5-fold), and Smad7 (2-fold) mRNA (Figure 6A). Although previous studies have implicated Thy-1 (36) and SMAD7 (37) in the blockade of TGF- $\beta 1$ responses in tissue fibroblasts (3842), the association of MSLN and MUC16 with Thy-1 and SMAD7 and their role in fibrogenesis has not been reported.

$M s l n$ is a part of a TGFBRI signaling complex. To explore the relationship between MSLN (31), Thy-1 (43), SMAD7 (44), and MUC16 (33), we performed a series of IPs using WT and $M s n^{-1}$ aPFs, with or without TGF- $\beta 1$ (Figure $6, \mathrm{C}-\mathrm{E}$ ). IP with anti-TGF $\beta$ RI $\mathrm{Ab}$ identified Thy-1, MUC16, and TGF $\beta$ RII proteins as binding partners of TGF $\beta$ RI in both WT and $M s n^{-1-}$ aPFs (Figure 6C). Interaction between TGF $\beta$ RI, Thy-1, and MUC16 was reduced in TGF- $\beta 1$-stimulated WT and $M s n^{-/}$aPFs, while binding of TGF $\beta$ RI to TGFRII was increased. In comparison with the WT aPFs, we observed that formation of a Thy-1-SMAD7-TGF $\beta$ RI complex was increased in unstimulated $M s n^{-/-}$aPFs, while TGFBRI-MUC16 binding was reduced and normalized to the amount of precipitated TGF $\beta$ RI (Figure 6E and Supplemental Figure 6A).
IP with anti-MSLN Ab (Figure 6D and Supplemental Figure 6B) revealed that MSLN binds to Thy-1 and MUC16 (but not to TGF $\beta$ RI or TGF $\beta$ RII) in WT aPFs. TGF- $\beta 1$ stimulation further increased the formation of an MSLN-Thy-1 complex, while MSLNMUC16 binding declined. As expected, we found that these complexes were absent in $\mathrm{Msln}^{-/-}$aPFs.

Finally, IP with anti-MUC16 Ab (Figure 6E and Supplemental Figure 6C) confirmed that MUC16 interacts with MSLN and TGFßRI (but not with Thy-1 or TGFßRII). We found that SMAD7 was also coprecipitated as a part of the MUC16-TGFßRI complex and that its interaction with MUC16 was decreased in response to TGF-1. We found that the formation of a MUC16-SMAD7 complex was increased in unstimulated $\mathrm{Msln}^{-/-} \mathrm{aPFs}$, while MUC16-TGF $\beta \mathrm{RI}$ binding was decreased, and detected no MUC16-MSLN complex. Taken together, we identified what we believe to be a novel TGF- $\beta 1$ signaling pathway, in which the activity of the TGFßRI-TGFßRII complex is regulated via interaction with a Thy-1-MSLN-MUC16SMAD7 complex, and in which MSLN and MUC16 facilitate TGF- $\beta 1$ responses in aPFs, while Thy- 1 and SMAD7 inhibit TGF- $\beta 1$ responses. Our proposed model of MSLN-dependent regulation of TGF- $\beta 1$ signaling in aPFs is depicted in Figure 6F.

$B D L$-induced liver fibrosis is reduced in $\mathrm{Muc16}^{-/-}$mice, but exacerbated in Thy-1/- mice. To further corroborate the findings on the in vivo role of MSLN, MUC16, and Thy-1 in cholestatic liver injury, we compared the development of BDL liver fibrosis in mice in which individual components of this signaling complex were knocked out. The role of MUC16-MSLN interaction in cholestatic fibrosis was evaluated in vivo in $\mathrm{Mucl6}^{-/-}, \mathrm{Muc16}^{-/-} \mathrm{Msln}^{-/}$, and $M \operatorname{snn}^{-1-}$ mice. Liver fibrosis induced by 5 days of BDL was equally attenuated in $\mathrm{Mucl6}^{-/-}, \mathrm{Mucl6}^{-/-} \mathrm{Msln} \mathrm{n}^{-/}$, and $\mathrm{Msln} \mathrm{n}^{-/-}$ mice (Figure 7A and Supplemental Figure 7), as shown by the reduced Picrosirius red-stained area and the downregulation of Col1a1, $\alpha$-SMA, and Timp1 mRNA compared with WT mice (Figure 7B), suggesting that MSLN and MUC16 regulate the same signaling pathway. Furthermore, inhibition of BDL-induced fibrosis in $\mathrm{Muc16}^{-/-} \mathrm{Msln}^{-/-}$mice (by 50\%) was comparable to that observed in aPF-ablated $M s \ln ^{\text {DTA }}$ mice (Figure 3), indicating that the MSLN/MUC16 pathway might be critical for aPF/myofibroblast activation. Therefore, we studied TGF- $\beta 1$-mediated activation of PFs in vivo in $M s l^{4 S m a d 4}$ mice (generated by crossing $M s l n^{n L a c Z-E R-C r e}$ and Smad4fl/fl mice) and in WT Msln ${ }^{n L a c Z-E R-C r e}$ mice (Figure 8A). Deletion of Smad4 in $\mathrm{MSLN}^{+}$aPFs attenuated BDL-induced (5 days) liver fibrosis by approximately 25\%, suggesting that MSLN-MUC16 functions in aPFs are not limited to regulation of the TGF- $\beta 1$ signaling pathway. We also examined the contribution of Thy- 1 to PF activation (Figure $8 \mathrm{~B}$ ) and found that deletion of Thy-1 in aPFs exacerbated cholestatic fibrosis in $T h y-1^{-/-}$mice by nearly $25 \%$ compared with that observed in WT mice, confirming that Thy-1 inhibits fibrosis. Overall, deletion of MSLN/MUC16 signaling inhibits the development of BDL-induced fibrosis by approximately $50 \%$, but only about $25 \%$ of this phenotype can be attributed to impaired TGF- $\beta 1$ signaling (as demonstrated by deletion of Smad4 specifically in $\mathrm{MSLN}^{+}$aPFs) and linked to the MSLN-MUC16-Thy-1 complex (since an increase of approximately $25 \%$ in liver fibrosis was detected in BDL-injured Thy-1//- mice). We concluded that MSLN/MUC16 pathway functions are not limited to regulation 
A

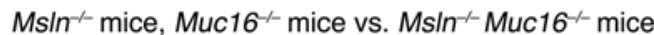

\begin{tabular}{|c|c|c|}
\hline \multicolumn{3}{|c|}{ No injury } \\
\hline WT & Muc16 ${ }^{--}$ & $\mathrm{Msln}^{-1-} \mathrm{Muc16}^{--}$ \\
\hline Picrosirius rec & & \\
\hline$\times 4$ & & \\
\hline
\end{tabular}
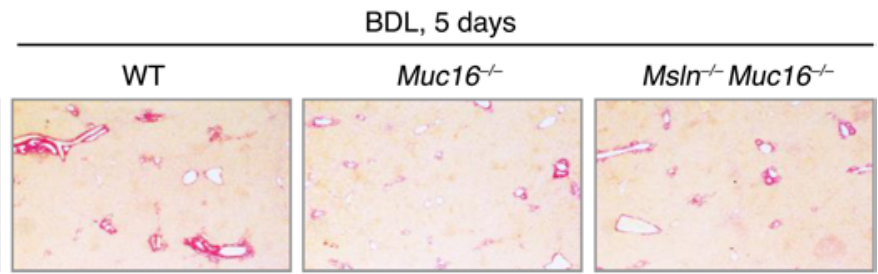

B
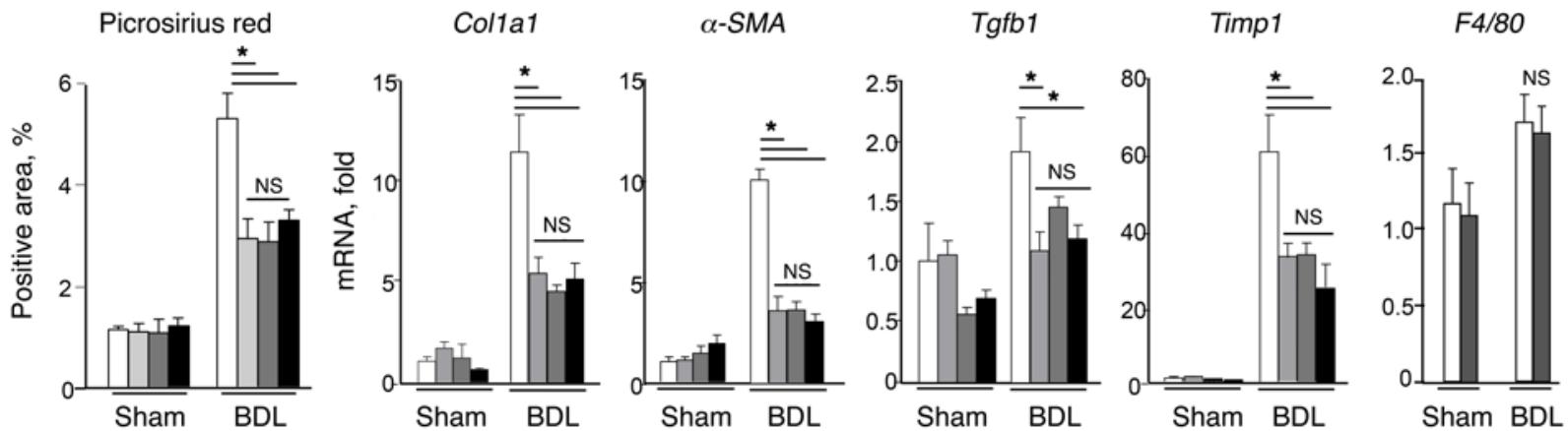

WT

$M s / n^{-1}$

Muc16

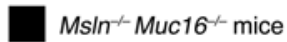

Figure 7. MSLN/MUC16 signaling regulates activation of aPFs/myofibroblasts in BDL-injured mice. (A) Liver fibrosis was compared in 8-week-old male BDL-operated (for 5 days) Ms/n $n^{-/-}$mice $(n=10)$, Muc16 ${ }^{-/-}$mice $(n=10), \mathrm{Ms}^{\prime-1-}$ Muc16 ${ }^{-/-}$mice $(n=9)$, and WT littermates $(n=5)$ (see also Supplemental Figure 7). Micrographs show Picrosirius red staining (original magnification, $\times 4$ ). (B) Data indicate the percentage of positively stained area. mRNA expression of fibrogenic, inflammatory, and aPF-specific genes was quantified by qPCR, and the data indicate the fold induction versus sham-operated WT mice. ${ }^{*} P<0.05$, by 2 -tailed Student's $t$ test

of TGF- $\beta 1$ signaling in aPFs and that the MSLN-MUC16 complex might further facilitate cholestatic fibrosis (by an additional 25\%, approximately) through the mediation of other important signaling pathways in aPFs. In the next set of experiments, we investigated the role of the MSLN/MUC16 signaling pathway in aPF proliferation.

Msln ${ }^{-/}$aPFs exhibit defective FGF-induced proliferation. FGF was identified as an important factor that drives the proliferation of aPFs. Primary cultured WT aPFs had a growth advantage over $\mathrm{Msln}^{-/-}$aPFs (Figure 5, B and C). In an attempt to further characterize MSLN-MUC16 functions, we examined the proliferation and responses to FGF using immortalized WT and $M s l n^{-/}$aPFs and found that cyclin D mRNA expression was suppressed by 4.5 -fold in FGF-stimulated $\mathrm{Msln}^{-/-}$aPFs and correlated with the downregulation of Mmp9 mRNA (Figure 9A). When we examined the components of the FGF signaling pathway, we found that JAK2 expression was increased by up to 4 -fold, while phosphorylation of JAK2-STAT3 was reduced ( $\downarrow 2$-fold) in FGF-stimulated $\mathrm{Msln}^{-/-}$aPFs (Figure 9B). Most strikingly, we observed that phosphorylation of AKT was strongly reduced (by 5-fold) in FGF-stimulated $\mathrm{Ms}^{-1 /-}$ aPFs (Figure 9C). A novel mechanism for terminating RTK signaling and reducing receptor abundance has been described and showed that, in AKTimpaired cells, RTK expression and trafficking are defective, resulting in prolonged signaling and increased activation of ERK1/2 (45). In accordance with previously published reports, we detected an 11-fold increase in ERK1/2 phosphorylation in FGF-stimulated $\mathrm{Msln}^{-/-}$aPFs (Figure 9B) that was associated with reduced degradation of FGF receptor 1 (FGFR1) following FGF signaling (Figure 9C). Next, we performed IPs with anti-FGFR1 Ab and anti-MSLN Ab to determine the relationship between MSLN and FGFR1, but did not identify MSLNMUC16 as binding partners of the FGFR1 complex (Figure 9D), suggesting that MSLN directly regulates AKT rather than the FGF signaling pathway.

Activation of JAK2-STAT3 and ERK1/2 regulates the proliferation of aPFs. We further investigated the molecular mechanism of FGF-mediated aPF proliferation (Figure 10 and Supplemental Figure 8). As expected, we found that pretreatment with an inhibitor of FGFR1 completely blocked (100\%, vs. DMSO) cyclin D mRNA expression in FGF-induced WT aPFs (Supplemental Figure 8). Cyclin D expression (Supplemental Figure 8) and STAT3 phosphorylation (Figure 10A) were also suppressed by inhibitors of AKT and JAK2, but most effectively (>80\%) by AZD1480, an inhibitor of JAK2 and ERK (Figure 10B). In agreement with these results, the proliferation and migration of WT and $M s n^{--}$aPFs to the scratch area were completely blocked by AZD1480 (Figure 10C) and associated with complete inhibition of ERK1/2 and STAT3 phosphorylation (Figure 10, B and C). Our proposed model of MSLN-MUC16-dependent regulation of FGF signaling in aPFs is depicted in Figure 10D.

Reconstitution of MSLN rescues the phenotype in $\mathrm{Msln}^{-/-}$cells. To confirm these findings, we transfected $\mathrm{Msln}^{-/-}$aPFs with a pCMV-MSLN construct (Figure 11A and Supplemental Figure 9A). We observed that reexpression of MSLN restored basal and FGF-dependent proliferation in $M s \ln n^{K O+M s l n}$ aPFs, as shown by the 
A

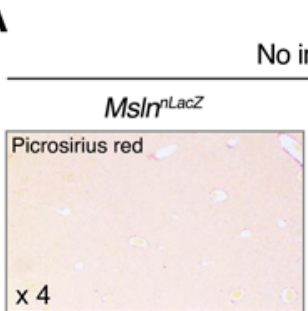

$M S I n^{\text {ILacZ-ER-Cre }}$ mice $\times$ Smad4 ${ }^{\text {fl/f }}$ mice

$M s / n^{4 S \operatorname{mad} 4}$
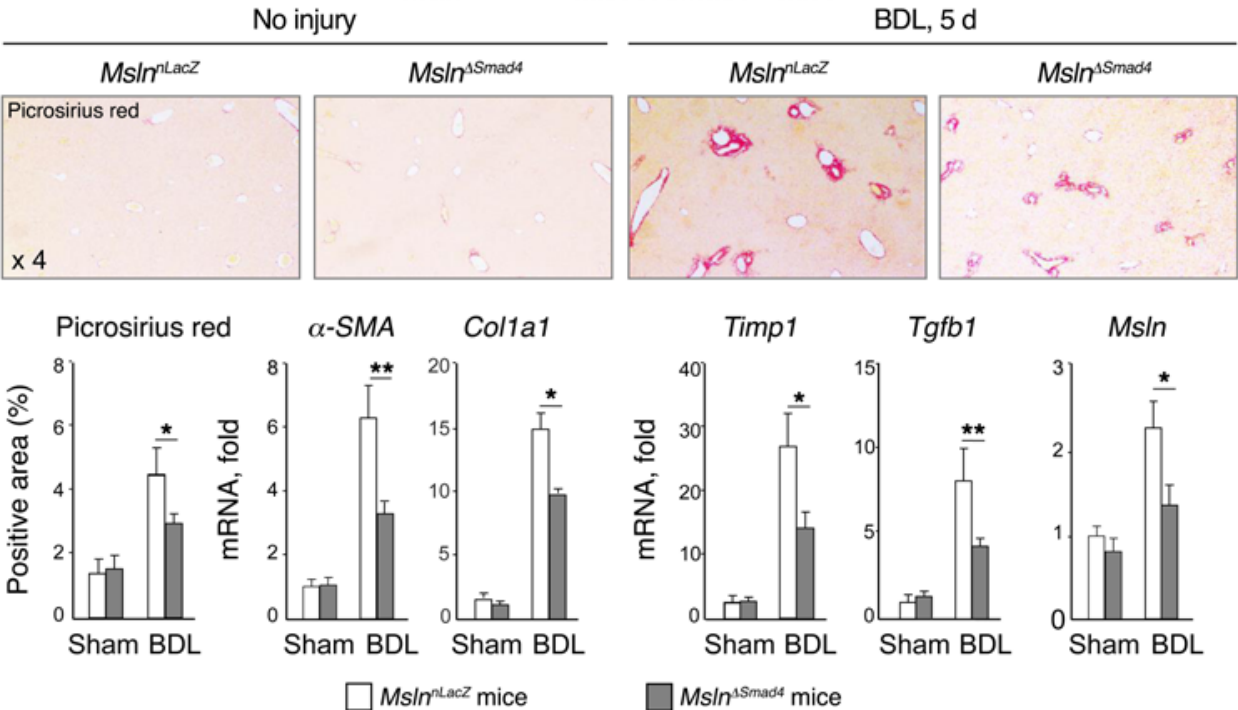

$M s I n^{n L a c Z}$ mice

B No injury
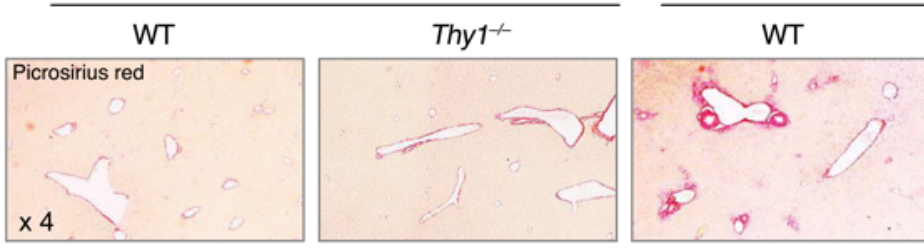

$\mathrm{BDL}, 5 \mathrm{~d}$
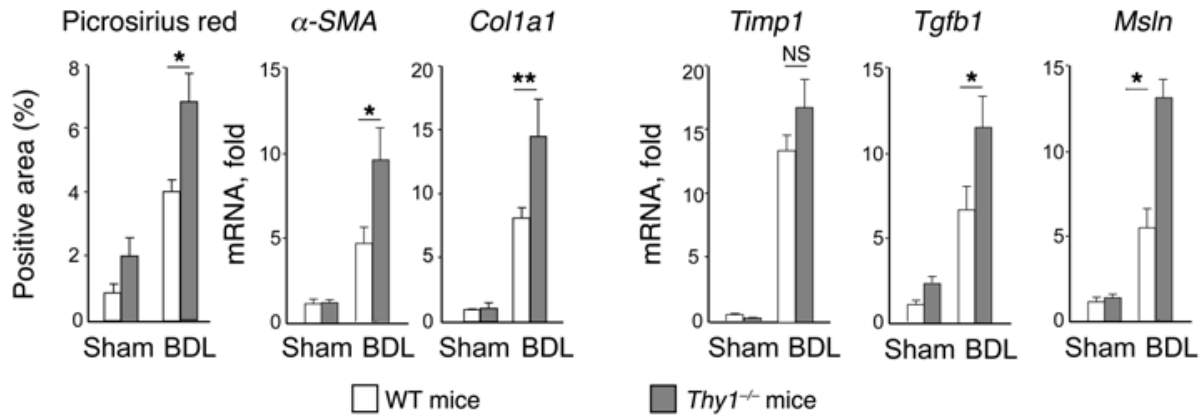

WT mice

Figure 8. Liver fibrosis is exacerbated in BDL-injured Thy-1/- mice. (A) Liver fibrosis was compared in 8-week-old male BDL-injured $M s / n^{45 m a d 4}$ mice and WT MsInnLacz littermates ( $n=9 /$ group). (B) Liver fibrosis was compared in 8-week-old male BDL-operated (for 5 days) WT and Thy-1 ${ }^{-/-}$littermates (C57BL/6, $n=8$ /group). (A and B) Micrographs show Picrosirius red staining (original magnification, $\times 4$ ). Data indicate the percentage of positively stained area. Expression of fibrogenic, inflammatory, and aPF-specific genes was quantified by qPCR, and the data indicate the fold induction versus sham-operated WT mice. ${ }^{*} P<0.01$ and ${ }^{*} P<0.05$, by 2 -tailed Student's $t$ test.

upregulation of cyclin D mRNA (Figure 11B) and the increased numbers of cells populating the scratch area (Figure 11C and Supplemental Figure 9B), both of which could be also blocked by AZD1480 (Supplemental Figure 9C). We found that reexpression of MSLN also restored Col1a1 and Muc16 in Msln ${ }^{K O+M s l n}$ aPFs, but had no effect on the expression levels of Thy-1, which remained elevated, as observed in Msln ${ }^{-1}$ aPFs (Figure 11A).

Inhibition of Thy-1 restores TGF- $\beta 1$-induced responses in $\mathrm{Msln}^{-1}$ cells. To test whether suppression of Thy-1 can restore the responsiveness of $M s l n^{--}$aPFs to TGF- $\beta 1$, we transfected $M s l^{-1}$ aPFs with Thy-1 siRNA or control siRNA (Figure 12A). Inhibition of Thy-1 restored phosphorylation of SMAD2 (Figure 9D) and the expression of Col1a1 in TGF- $\beta 1$-stimulated Msl${ }^{K O+s i T h y-1}$ aPFs, but had no effect on cyclin D (Figure 12, A and B) or ERK1/2 (Supplemental Figure 10), suggesting that the regulatory function of Thy-1 is restricted to TGF- 1 signaling.

Overexpression of AKT restores $\mathrm{Msln}^{-1-}$ cell proliferation. Furthermore, to test whether restoration of AKT phosphorylation can reinstate the proliferation of $\mathrm{Msln}^{--}$aPFs, we infected $\mathrm{Msln}^{-/}$aPFs with constitutively activated (CA) AKT (AAV-CA-AKT) or with a control adenoviral construct expressing GFP (AAV-GFP). We found that expression of cyclin D and cyclin E (Figure 13A) and FGF-induced degradation of FGFR1 were restored in $M s h^{K O-A A V-C A-A K T}$ aPFs to the levels observed in WT aPFs (Figure 13B). The changes in JAK2 and its phosphorylation observed in MSLN-deficient aPFs were restored to normal levels in $M s l{ }^{K O-A A V-C A-A K T}$ aPFs (Figure 13B and 
A

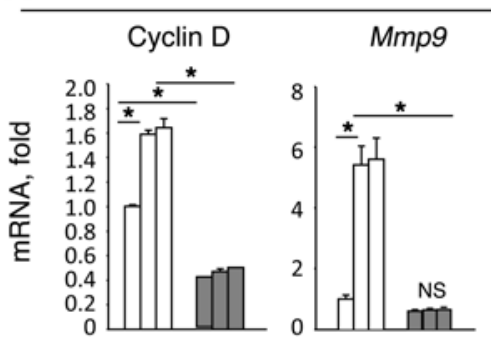

FGF, 24h $012 \quad 012 \mathrm{ng} / \mathrm{ml} \quad 012 \quad 012 \mathrm{ng} / \mathrm{ml}$
Immortalized aPFs

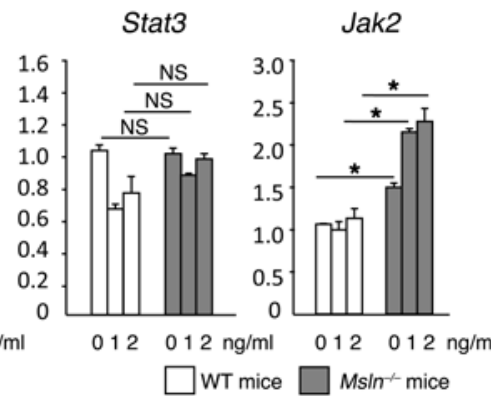

B

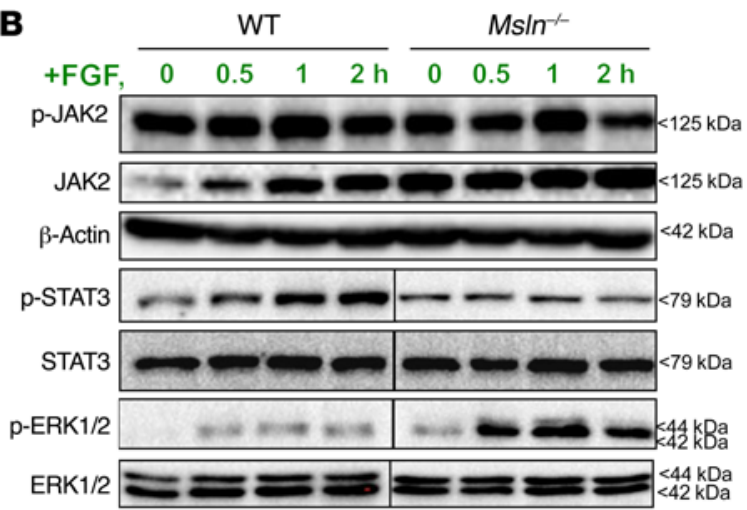

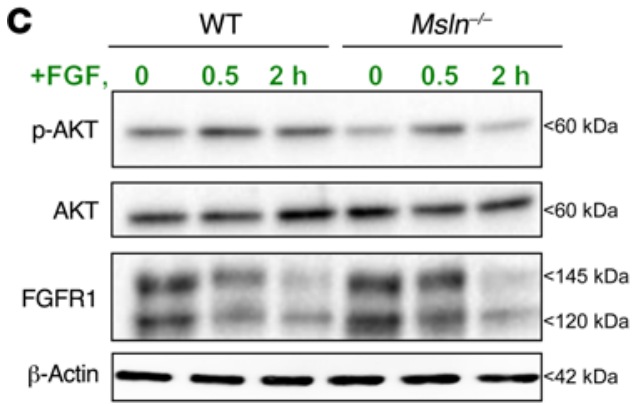

D

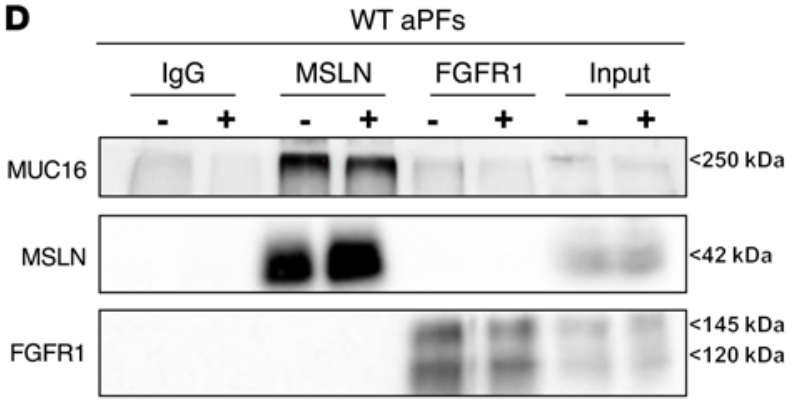

Figure 9. Ms/n ${ }^{-1-}$ aPFs exhibit a defect in FGF signaling. (A) Responses to FGF (2 ng/ml) were compared in immortalized WT and Ms/n ${ }^{-/-}$aPFs using qPCR. ${ }^{*} P<0.05$, by ANOVA. Expression of JAK2, STAT3, ERK1/2 (B) and AKT and FGFR1 (C) was analyzed in FGF-stimulated immortalized WT and Ms/n ${ }^{-1-}$ aPFs using immunoblotting, normalized to $\beta$-actin protein expression. Representative immunoblots (from $>3$ independent experiments) are shown. (D) Cell lysates of WT aPFs exposed to vehicle or FGF were immunoprecipitated with Abs targeting TGF $\beta R I$, MSLN, FGFR1, or control IgG. The immunoprecipitates were probed for expression of MUC16, MSLN, and FGFR1. Representative immunoblots of more than 3 independent IPs are shown.

Supplemental Figure 11A), while activation of ERK1/2 remained unchanged (Supplemental Figure 11B). Furthermore, we found that TGF- $\beta 1$-mediated phosphorylation of SMAD2 was not rescued in Ms $\ln ^{K O-A A V-C A-A K T}$ aPFs (Supplemental Figure 11C), suggesting that activation of AKT does not affect TGF- $\beta 1$ signaling pathway in aPFs.

\section{Discussion}

We demonstrate here that aPFs mediate hepatic fibrosis at the onset of cholestatic injury and that ablation of $\mathrm{MSLN}^{+} \mathrm{aPFs}$ in mice decreases BDL-induced ( 5 days) liver fibrosis by approximately $50 \%$. This study links MSLN/MUC16 signaling to the pathogenesis of cholestatic fibrosis and shows that deletion of MSLN and/ or MUC16 in mice reduces cholestatic liver fibrosis by nearly $50 \%$. We determined that MSLN facilitates both TGF- $\beta 1$-induced activation of aPFs and FGF-induced proliferation of aPFs. We detected a similar upregulation of $\mathrm{MSLN}^{+}$aPFs in patients with biliary fibrosis of different etiologies. Our data demonstrate that therapeutic administration of MSLN-blocking Abs attenuates BDLinduced liver fibrosis in mice, suggesting that MSLN could serve as a target for antifibrotic therapy.

MSLN (19) is highly expressed during embryonic development $(17,32)$ but is downregulated in adulthood $(20)$. However, $M s h^{-1-}$ and $\mathrm{Mucl6}^{-/-}$mice do not have developmental abnormalities $(31,33)$. In turn, human MSLN and CA125 (the human counterpart of MUC16) is highly upregulated in several malignancies such as ovarian cancer, hepatocellular carcinoma, and mesothe- lioma (26), and both MSLN and CA125 became targets for anticancer therapy. Here, we demonstrate that expression of MSLN is not restricted to tumorigenesis, but is strongly induced in aPFs in response to cholestatic liver injury (Figure 1 and Supplemental Figure 1A). In support of this, $M s \ln$-KO mice developed a defect in the activation of cancer-associated myofibroblasts that resulted in reduced tumorigenesis (25-29).

Despite extensive studies of human cancer cells, the functional properties of the MSLN/MUC16 signaling pathway remain poorly understood. MUC16 was identified as an MSLN ligand, and MSLN/MUC16 signaling was linked to AKT and ERK1/2 activation and was implicated in the metastatic growth and dissemination of cancer cells (46-49). Like other GPI-anchored proteins, MSLN requires MUC16 and/or utilizes other receptors for intracellular signaling. Our studies of immortalized aPFs show that $M s l^{-1-}$ aPFs exhibit impaired proliferation and responses to FGF and that these defects are associated with defective AKT phosphorylation (Figure 9C). However, co-IP experiments did not detect MSLN-MUC16 as a part of the FGF/ FGFR1 signaling complex. A novel mechanism of AKT activation has been identified that implicates AKT in the regulation of RTK trafficking and degradation (45). Accordingly, cells deficient in AKT activation fail to properly express EGFR and PDGFR, but as a compensatory mechanism, strongly upregulate ERK1/2 phosphorylation. We observed a similar phenotype in $M s n^{-1}$ aPFs (Figure 9B). The loss of AKT activation was asso- 
A

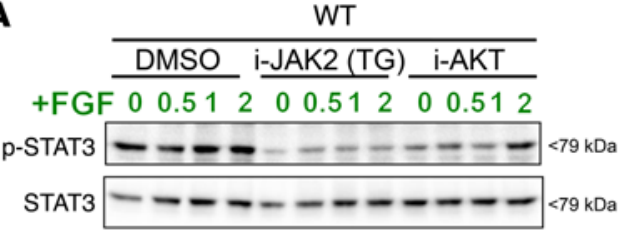

B

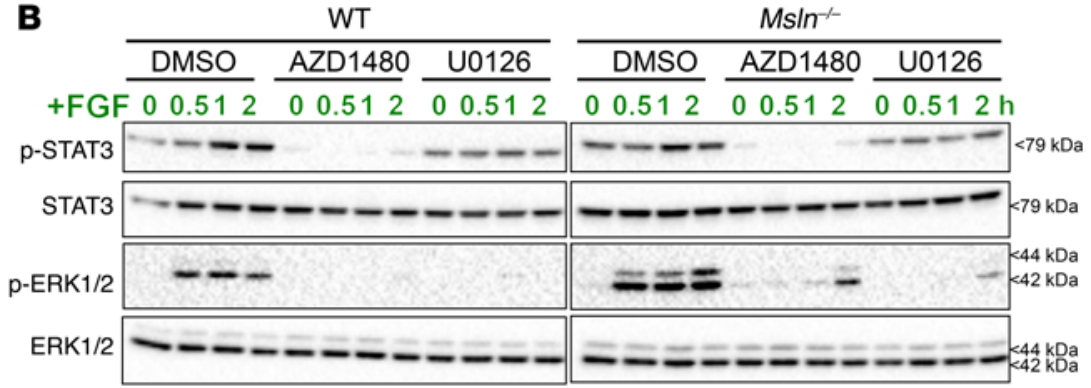

C

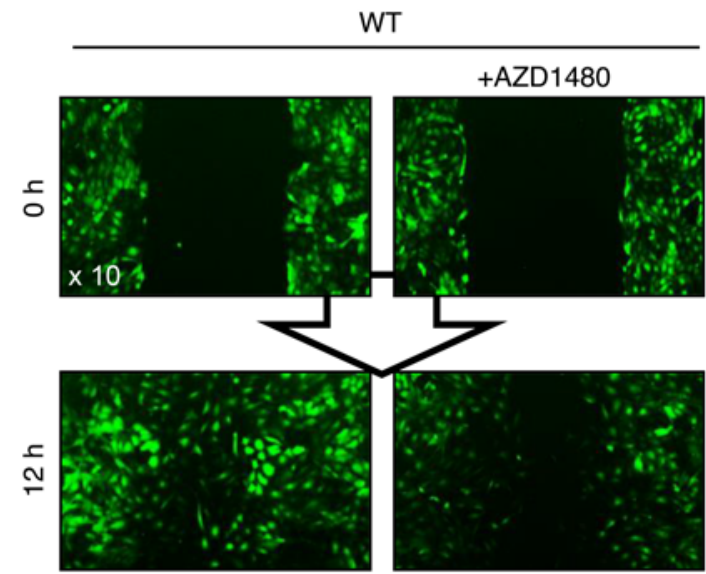

D

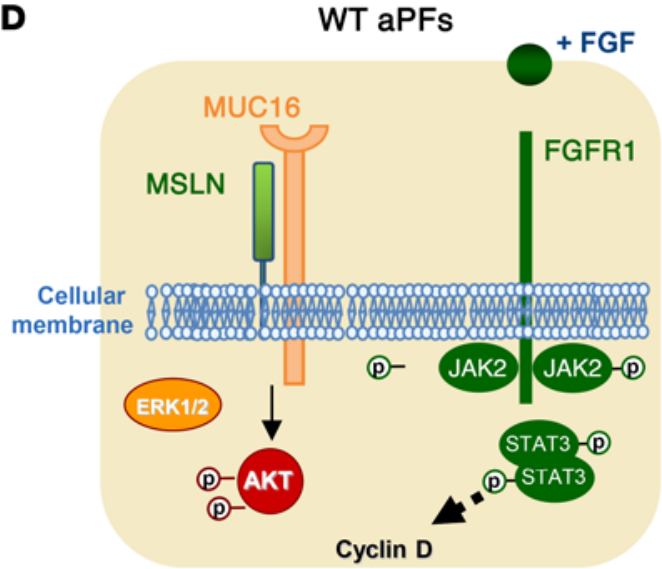

$M \operatorname{Mln} n^{--}$
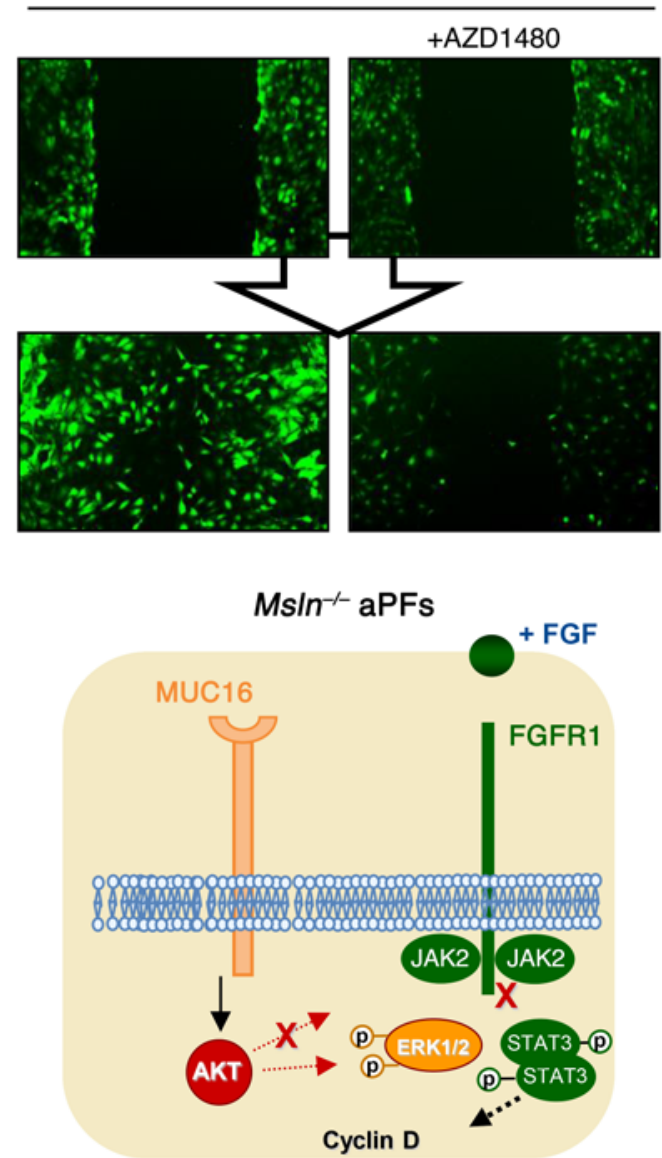

Figure 10. Regulation of FGF signaling pathways in WT aPFs. (A) Immortalized WT and Ms/n ${ }^{-1-}$ aPFs (105/well) were pretreated for 20 minutes with DMSO, inhibitor-JAK2 (i-JAK2) (TG-101348, $1 \mu \mathrm{M})$, or inhibitor-AKT (i-AKT) $(0.5 \mu \mathrm{M})$, or with (B) AZD1480 $(1 \mu \mathrm{M})$ or U0126 (5 $\mu \mathrm{M})$ and then stimulated with or without FGF $(2 \mathrm{ng} / \mathrm{ml})$. Representative immunoblots are shown in A and B. (C) WT and Ms/n ${ }^{-1-} \mathrm{aPFs}\left(10^{5} /\right.$ well) were pretreated for 20 minutes with DMSO or AZD1480 $(1 \mu \mathrm{M})$ and subjected to a 12-hour scratch assay. Proliferation and migration of WT and Ms/n ${ }^{-/-}$aPFs were blocked by AZD1480. Representative fluorescent (Col-GFP) images of more than 3 independent experiments are shown (original magnification, $\times 10$ ). (D) Proposed model of MSLN regulation of FGF signaling. Binding of FGF to FGFR1 results in activation of the JAK2/STAT3 signaling pathway, upregulation of cyclin D, and proliferation of WT aPFs. Subsequently, phosphorylation ( $p$ ) of AKT is required for the degradation of FGFR1 in WT aPFs. In turn, Ms/n ${ }^{--}$aPFs exhibit a defect in AKT phosphorylation, which results in compensatory phosphorylation of ERK1/2 and a failure to properly express and degrade FGFR1. In addition, FGF-inducible activation of JAK2 and STAT3 is impaired in Ms/n ${ }^{-1-}$ aPFs, and basal proliferation of Ms/n ${ }^{-1-}$ aPFs is mediated by ERK1/2-dependent phosphorylation of STAT3. Overall, the MSLN/MUC16/AKT pathway is a key regulator of cholestasis-induced proliferation of WT aPFs/myofibroblasts. The loss of AKT activation is associated with an inability of $\mathrm{Ms}_{\mathrm{I}} \mathrm{n}^{-/} \mathrm{aPFs}$ to mount FGF-mediated responses (see also Supplemental Figure 8).

ciated with an inability of $M s \mathrm{In}^{-/-}$aPFs to mount FGF-mediated responses. Because of reduced phosphorylation of JAK2 (despite upregulation of JAK2 expression), we observed that FGF-dependent activation of STAT3 was also impaired in $\mathrm{Msln}^{-/-}$aPFs. In turn, we observed that basal and FGF-induced ERK1/2 activation was highly increased in $M s{ }^{-/-}$aPFs and was driving the residual proliferative capability of $\mathrm{Msln}^{-/-}$aPFs (via phosphorylation of STAT3). Although the exact mechanism by which MSLNMUC16 activates AKT remains unknown, our data indicate that the MSLN/MUC16/AKT pathway is a key regulator of cholestasis-induced proliferation of aPFs/myofibroblasts, but does not affect the fibrogenic properties of aPFs. 
A

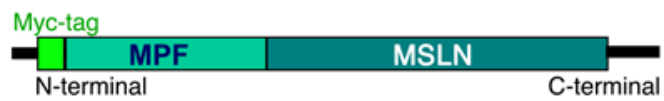

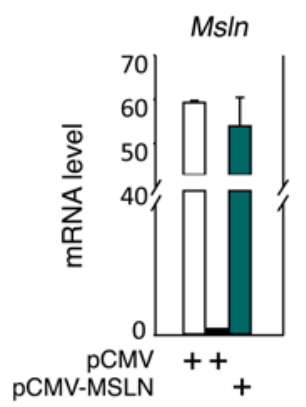

B

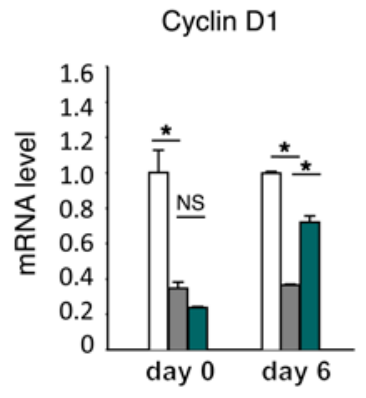

Col1a1
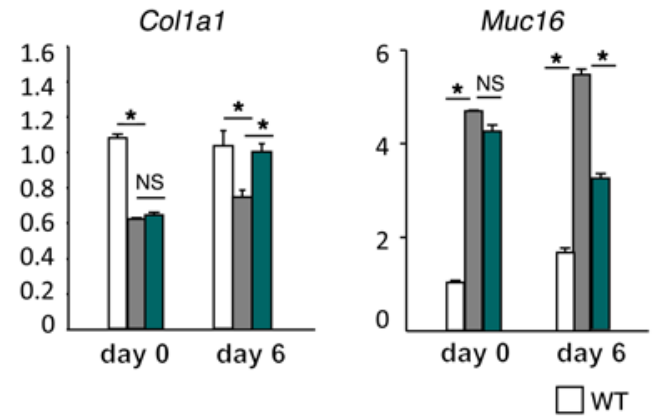

Thy-1

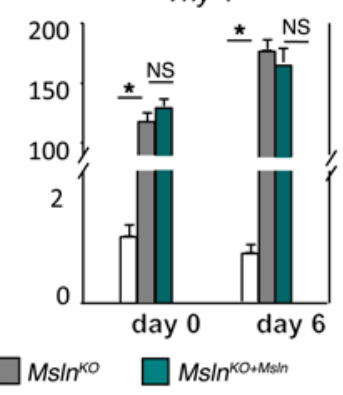

C $\left.{ }^{100}\right]$

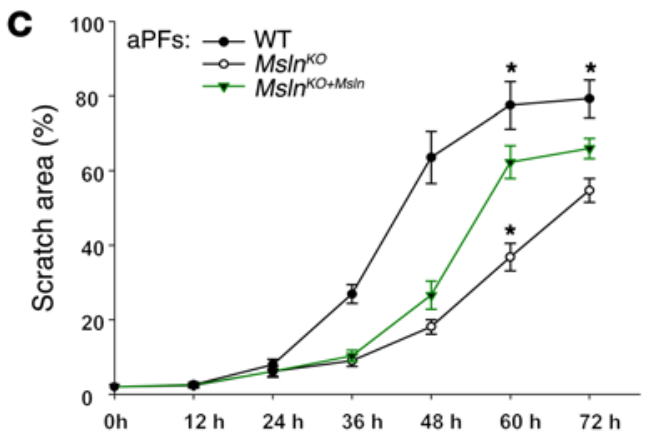

Figure 11. MSLN/MUC16 signaling regulates Thy-1/TGFßRI and AKT/FGFR1 pathways in aPFs. (A-C) Ms/n cDNA was cloned into a pCMV-Tag3A expression vector and transfected into Ms $/ n^{---}\left(M s / n^{K O+M s / n}\right)$ aPFs. MSLN-Myc fusion protein expression in Ms/n ${ }^{-1-}$ aPFs was detected by qPCR (immunostaining with anti-Myc Ab is shown in Supplemental Figure 9A). (A) Expression of Msln, Col1a1, Muc16, Thy-1, and (B) cyclin D mRNA was analyzed in Ms/n ${ }^{K 0+M s i n}$ aPFs. (C) WT, Ms $/ n^{K O+M s l n}$, and $M s / n^{-1-}$ aPFs were replated 72 hours after transfection (10 $/$ well) and subjected to a scratch assay (see Supplemental Figure 9 , B and $\mathrm{C}$ ). The scratch area was calculated as a percentage and represents the average of more than 3 experiments. ${ }^{*} P<0.05$, by ANOVA.

$M s n^{-1}$ aPFs exhibit defective TGF- $\beta 1$ signaling manifested by reduced expression of profibrogenic genes and TGF- $\beta 1$ target genes (Figure 6A). Here, we demonstrate the involvement of MSLN in the TGF- $\beta 1$ signaling pathway and propose a mechanism in which the MSLN-MUC16 complex interacts with the TGFBRI receptor and facilitates TGF- $\beta 1$ signaling in WT aPFs via disruption of TGF $\beta$ RI binding to its inhibitory receptor Thy-1 (Figure 6, $\mathrm{C}$ and D). This enables TGF $\beta$ RI-TGF $\beta$ RII interaction, phosphorylation and nuclear translocation of SMAD2/3/4, and transcription of target genes. In turn, deletion of Msln in Msln ${ }^{-1}$ aPFs resulted in overexpression of Thy-1 (>150-fold vs. WT levels), which binds to TGF $\beta R I$ and prevents TGF- $\beta 1$ signaling. Consistently, inhibition of Thy- 1 partially restored TGF- $\beta 1$ signaling in $M s n^{-1-}$ aPFs, but had no effect on ERK1/2 activation.

Like MSLN, Thy-1 is a GPI-anchored protein (50) that is expressed in fibroblasts, $\mathrm{T}$ cells, and neurons and is considered a specific marker for these cell types. Thy- 1 was implicated in the inhibition of TGF- $\beta 1$ responses in tissue fibroblasts via interaction with $\alpha v-\beta 5$ integrins (38-42). While Thy- 1 functions are not well characterized, deletion of $T h y-1$ in mice was shown to exacerbate bleomycin-induced lung fibrosis (36). Our data suggest that Thy-1 blocks TGFRRI and that formation of the MSLN-Thy-1 complex enables TGF- $\beta 1$ signaling. In accordance with this, downregulation of Thy-1 expression restored TGF- $\beta 1$ signaling in $\mathrm{Msln}^{-/-}$mice, and deletion of Thy-1 in mice aggravated the development of cholestatic liver fibrosis. We identified TGF $\beta R I$ as a binding partner of Thy- 1 and MUC16 as a binding partner of MSLN. Since knocking out MSLN and MUC16 receptors together or separately yielded similar phenotypes in BDL-injured mice (Figure 7), we propose that the MSLN-MUC16 complex regulates the same signaling pathway in tissue fibroblasts and, therefore, can serve as a unique target for antifibrotic therapy in patients with cholestatic fibrosis.

Overall, our proposed model (summarized in Figure 6F and Figure 10D) suggests that the MSLN/MUC16 signaling pathway facilitates cholestatic fibrosis in mice via regulation of TGF- $\beta 1-$ mediated activation and FGF-induced proliferation of aPFs. In response to TGF- $\beta 1$ signaling, MSLN disrupts the Thy-1-TGF $\beta$ RI inhibitory complex and enables binding of TGF- $\beta 1$ to its cognate receptor, phosphorylation and nuclear translocation of SMAD2/3, and transcription of the TGF- $\beta 1$ target genes PAI1 and activin. MSLN and Thy- 1 differentially regulate TGF- $\beta 1$ signaling in aPFs, and MSLN serves as a functional repressor of Thy-1. This represents, to our knowledge, a novel level of regulation of TGF- $\beta 1$ signaling that may have broad implications for tissue homeostasis, remodeling, and fibrosis in multiple organs. Furthermore, our data suggest that the MSLN-MUC16 complex regulates FGF-dependent proliferation of aPFs, although indirectly, via activation of AKT, which regulates the trafficking and degradation of FGFR1 (45). 
A

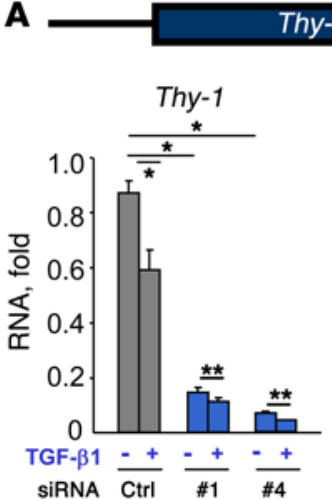

Col1a1

aPFs:

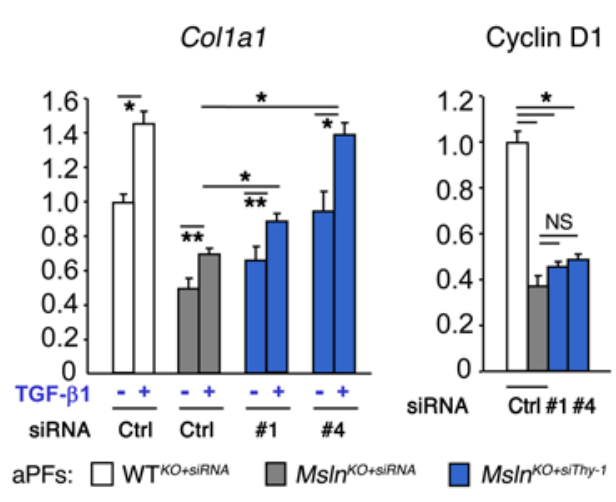

B

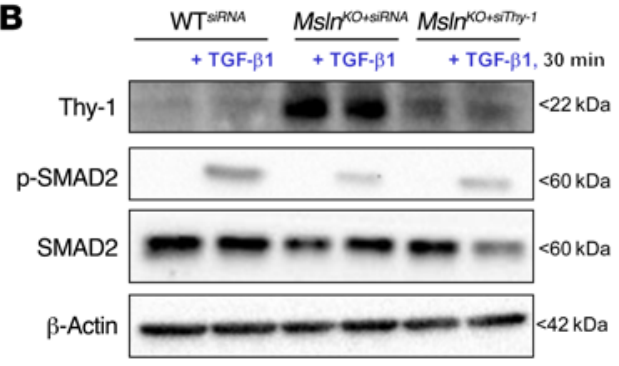

Figure 12. Thy-1 regulates TGF- $\beta 1$ pathways in aPFs. (A and $\mathbf{B}$ ) $M s / n^{-1-}$ aPFs were transfected with control (Ctrl) or Thy-1 (nos. 1 and 4) siRNAs. (A) Expression of Thy-1, Col1a1, and cyclin D mRNA.

(B) Expression and phosphorylation of SMAD2, Thy-1, and $\beta$-actin were evaluated by immunoblotting (see also Supplemental Figure 10). ${ }^{*} P<0.05$ and ${ }^{* *} P<0.01$, by ANOVA. Representative immunoblots from more that 3 independent experiment are shown.

ers associated with the self-renewal capability of adult mesenchymal cells. Remarkably, expression of the same markers was detected in aPFs $(2,4,6)$. Given the high proliferative capacity of aPFs emerging in fibrosing liver within days after $\mathrm{BDL}$, we can speculate that aPFs might derive from hepatic mesothelial progenitors. Heterogeneity of the aPF/myofibroblast population cannot be excluded, and upregulation of appropriate markers in aPFs may vary, depending on the duration of BDL, the stage of activation/ differentiation, and the localization within the hepatic lobule. In support of this, expression of MSLN, Thy-1, and fibulin 2 is induced in aPFs throughout BDL injury (Figure 1). And in agreement with previous findings (34), we observed that mesothelial cells, identified by expression of the GPM6A marker, also contributed to a small population of $\mathrm{Col}^{+}$myofibroblasts residing in the liver capsule of WT mice that had undergone 5 days of BDL injury (Supplemental Figure 2), while the majority of myofibroblasts were composed of $\mathrm{Thy}^{-1}{ }^{+} \mathrm{Col}^{+}$aPFs/myofibroblasts, located in the portal areas, and desmin ${ }^{+} \mathrm{Col}^{+}$aHSCs/myofibroblasts, located in the acinus. Concordant with our previous findings, aPFs/ myofibroblasts contributed to $60 \%$ to $70 \%$ of hepatic myofibroblasts after BDL-induced (5 days) liver fibrosis (4).

\section{Methods}

Patient specimens. Archived liver specimens were obtained from 16 patients with liver cirrhosis (HCV, $n=5$ [METAVIR score of F2]; obstructive jaundice, $n=5$ (F2); BA, $n=6$ ) and from 3 patients who were undergoing liver resection for reasons unrelated to liver fibrosis (normal liver) at the Kyoto University Hospital (2010 to 2013).

Mice and liver injury. Eight-week-old collagen $\alpha 1(\mathrm{I})-\mathrm{GFP}$ mice (62), Msl ${ }^{-/}$mice (31), Msln ${ }^{\text {nLacZ-ER-Cre }}$ mice (53), Muc16 ${ }^{-1-}$ mice (33), Thy $-1^{-/-}$mice (50), Rosa26 flox-Stop-flox-DTA mice (The Jackson Laboratory; 009669), Smad flfl $^{\text {mice }}$ (The Jackson Laboratory; 017462), and WT littermates on a C57BL/6 background were maintained under specific pathogen-free conditions at UCSD and had ad libitum access to normal chow and water. Liver injury was induced in mice by ligation of the common bile duct (for 5 days and 17 days), or by oral gavage with $\mathrm{CCl} 4$ carbon tetrachloride $\mathrm{CCl}_{4}$ (1:4 dilution in corn oil, $60 \mu \mathrm{l} \times$ 14 injections) (4). An additional model of cholestatic liver fibrosis was induced in WT and $\mathrm{Msln}^{-/-}$mice fed a diethoxycarbonyl dihydrocollidine (DDC) diet (AD5001; Custom Animal Diets) or a control diet (AD3012; Custom Animal Diets) for 3 weeks.

Serum ALT and ALP measurements. Serum ALT and ALP measurements were analyzed using Infinity ALT (Thermo Fisher Scientific), a QuantiChrom ALP Assay Kit (BioAssay Systems), and a VALIDATE calibration verification kit (Maine Standards Company LLC).

Histology. Formalin-fixed, paraffin-embedded human or mouse livers were stained with Picrosirius red (analyzed by a pathologist)
(59), gremlin 1 (60), MSLN (53), CD9 (61), and CD105 (59) (and/or others), which are newly recognized progenitor mark- 
A
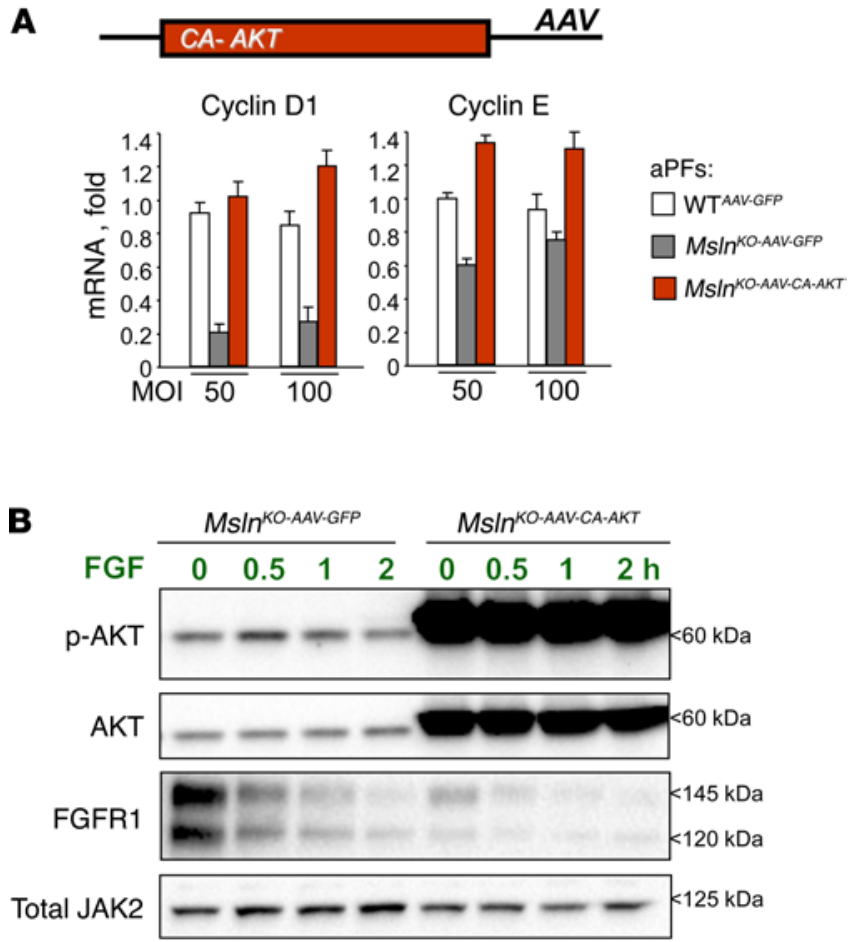

(4). Human livers were immunostained with anti-human $\alpha$-SMA (Dako; M0851); anti-human desmin (Thermo Fisher Scientific; PA5-16705); anti-human MSLN (Sigma-Aldrich; HPA017172); and anti-human Thy-1 (Abcam; ab92574) Abs. Mouse livers were stained with anti-mouse $\alpha$-SMA (Abcam: ab5694); anti-mouse desmin (Thermo Fisher Scientific: RB-9014-P0); and anti-mouse F4/80 (eBioscience: 14-4801-82) Abs, followed by staining with DAB (Vector Laboratories) or with secondary Alexa Fluor 594conjugated Abs. LacZ staining was performed in freshly isolated mouse livers (Sigma-Aldrich; GALS1KT). Images were taken using an Olympus microscope, and positive areas were calculated using ImageJ software (NIH) (see Supplemental Methods).

Cell culture. The RFL-6 CD90.2 cell line (rat lung Thy-1 $1^{+}$fibroblasts, gift of James S. Hagood, (UCSD, La Jolla, California, USA) and LMP cell line (murine pancreatic $\mathrm{MSLN}^{+}$and MUC16 ${ }^{+}$cancer, gift of Andrew M. Lowy, (UCSD, La Jolla, California, USA) were used for IP experiments. Reconstitution of MSLN was achieved in $M s l n^{-/-}$aPFs by transfection of pCMV-Tag3A-N-Myc-MSLN. Thy-1 knockdown was achieved in $M s n^{-/}$aPFs with Thy-1 siRNA (QIAGEN), transfected using a FlexiTube GeneSolution (GS21838). CA AKT was overexpressed in $M s n^{-/-}$aPFs infected with adenovirus AAV-CA-AKT expressing an amino-terminal myristylation signal (AdmyrAkt) (63), compared with control AAV-GFP (see Supplemental Methods).

qPCR. Total RNA was isolated from livers or isolated aPFs using RNeasy columns (QIAGEN) (4). Expression levels of selected genes were calculated after normalization to the standard housekeeping gene HPRT (Invitrogen, Thermo Fisher Scientific) using the $\Delta \Delta \mathrm{Ct}$ method. The data represent relative mRNA levels compared with control levels (mean \pm SEM, $P<0.05$ ).

IP and Western blot analysis. IPs from the precleared whole-cell lysates were performed at $4^{\circ} \mathrm{C}$ overnight using $2 \mu \mathrm{g}$ anti-MSLN Ab
Figure 13. MSLN/MUC16/AKT signaling regulates FGF-dependent proliferation of aPFs. (A and B) Ms/n $n^{---}$aPFs were infected with CA AKT (MsIn ${ }^{K O-A A V-C A-A K T}$ aPFs). (A) Expression of cyclin $D$ and cyclin $E$ was detected by qPCR. (B) Phosphorylation of JAK2 was restored in Ms/n $n^{K O-A A V-C A-A K T}$ aPFs and was normalized to JAK2 protein expression levels (see also Supplemental Figure 11). Data shown is representative of more that 3 independent experiments.

(IBL; catalog 28127), anti-TGFßRI Ab (Abcam; catalog aB31013), or anti-MUC16 Ab (Abicode; catalog R2334-3) and analyzed by Western blotting (see Supplemental Methods).

Statistics. All data represent the mean \pm SEM. Comparisons of 2 groups were analyzed using an unpaired, 2-tailed Student's $t$ test. Comparisons of 3 or more groups were analyzed using ANOVA. ANOVA with a Dunnett's test was used for comparing multiple groups of mice or treatments with controls. ANOVA with a Bonferroni's test was used when making multiple pair-wise comparisons between different groups. A $P$ value of less than 0.05 was considered statistically significant. Analyses were performed using GraphPad Prism (Prism 5 version 5.03; GraphPad Software).

Study approval. All mice were maintained under specific pathogen-free conditions at UCSD according to UCSD IACUC protocol S07088. For human subjects, written informed consent was obtained from each patient in accordance with the ethics guidelines for epidemiological research in Japan (E2077 protocol, approved by the Ethics Committee of Kyoto University Graduate School of Medicine).

\section{Author contributions}

YK performed in vivo experiments, analyzed the data, and wrote the manuscript. PW, SL, and DK performed in vitro experiments. $\mathrm{KI}$ performed in vivo experiments and conducted human studies. XL, JX, MZ, MS, and MC performed experiments. KT and TB provided expertise in liver fibrosis and mesothelin. $\mathrm{CB}$ and $\mathrm{SH}$ assisted with data analysis. DAB provided support and critical revision of the manuscript. TK provided support, designed the study, and wrote the manuscript.

\section{Acknowledgments}

We thank Gianfranco D. Alpini (Baylor Scott \& White Healthcare, Temple, Texas, USA) for providing mRNA from isolated cholangiocytes. We thank James S. Hagood (UCSD, La Jolla, California, USA) and Yuval Rinkevich (Institute of Lung Biology and Disease [iLBD] Helmholtz Zentrum München, Munich, Germany) for providing $T h y-1^{-/-}$and $M s{ }^{E R-C r e}$ mice. We are grateful to Koji Taniguchi (Keio University, Tokyo, Japan) for his technical support on confocal microscopy. We thank Karin Diggle (UCSD, La Jolla, California, USA) for her dedicated management of our laboratory. This research was supported by the NIH (AA022614, DK099205, DK101737, DK111866, AA011999, ES010337, AA021856, and GM041804); the Japanese Ministry of Health, Labour and Welfare; and the American Liver Foundation. This research was supported in part by the Intramural Research Program of the NIH, National Cancer Institute, Center for Cancer Research.

Address correspondence to: Tatiana Kisseleva, 9500 Gilman Drive, \#0063, La Jolla, California 92093, USA. Phone: 858.822.5339; E-mail: tkisseleva@ucsd.edu. 
1. Bataller R, Brenner DA. Liver fibrosis. J Clin Invest. 2005;115(2):209-218.

2. Dranoff JA, Wells RG. Portal fibroblasts: Underappreciated mediators of biliary fibrosis. Hepatology. 2010;51(4):1438-1444.

3. Kisseleva T, et al. Myofibroblasts revert to an inactive phenotype during regression of liver fibrosis. Proc Natl Acad Sci U S A. 2012;109(24):9448-9453.

4. Iwaisako K, et al. Origin of myofibroblasts in the fibrotic liver in mice. Proc Natl Acad Sci U S A. 2014;111(32):E3297-E3305.

5. Desmoulière A. Hepatic stellate cells: the only cells involved in liver fibrogenesis? A dogma challenged. Gastroenterology. 2007;132(5):2059-2062.

6. Wells RG. The portal fibroblast: not just a poor man's stellate cell. Gastroenterology. 2014;147(1):41-47.

7. Fausther M, Dranoff JA. Beyond scar formation: portal myofibroblast-mediated angiogenesis in the fibrotic liver. Hepatology. 2015;61(3):766-768.

8. Bhunchet E, Wake K. Role of mesenchymal cell populations in porcine serum-induced rat liver fibrosis. Hepatology. 1992;16(6):1452-1473.

9. Bosselut N, et al. Distinct proteomic features of two fibrogenic liver cell populations: hepatic stellate cells and portal myofibroblasts. Proteomics. 2010;10(5):1017-1028.

10. Desmoulière A, et al. Extracellular matrix deposition, lysyl oxidase expression, and myofibroblastic differentiation during the initial stages of cholestatic fibrosis in the rat. Lab Invest. 1997;76(6):765-778

11. Goodpaster T, Legesse-Miller A, Hameed MR, Aisner SC, Randolph-Habecker J, Coller HA. An immunohistochemical method for identifying fibroblasts in formalin-fixed, paraffin-embedded tissue. J Histochem Cytochem. 2008;56(4):347-358.

12. Lepreux S, Desmoulière A. Human liver myofibroblasts during development and diseases with a focus on portal (myo)fibroblasts. Front Physiol. 2015;6:173.

13. Tuchweber B, Desmoulière A, Bochaton-Piallat ML, Rubbia-Brandt L, Gabbiani G. Proliferation and phenotypic modulation of portal fibroblasts in the early stages of cholestatic fibrosis in the rat. Lab Invest. 1996;74(1):265-278.

14. Uchio K, Tuchweber B, Manabe N, Gabbiani G, Rosenbaum J, Desmoulière A. Cellular retinolbinding protein-1 expression and modulation during in vivo and in vitro myofibroblastic differentiation of rat hepatic stellate cells and portal fibroblasts. Lab Invest. 2002;82(5):619-628.

15. Wells RG, Kruglov E, Dranoff JA. Autocrine release of TGF- $\beta$ by portal fibroblasts regulates cell growth. FEBS Lett. 2004;559(1-3):107-110.

16. Dudas J, Mansuroglu T, Batusic D, Ramadori G. Thy- 1 is expressed in myofibroblasts but not found in hepatic stellate cells following liver injury. Histochem Cell Biol. 2009;131(1):115-127.

17. Knittel T, et al. Rat liver myofibroblasts and hepatic stellate cells: different cell populations of the fibroblast lineage with fibrogenic potential. Gastroenterology. 1999;117(5):1205-1221.

18. Dranoff JA, Kruglov EA, Robson SC, Braun N, Zimmermann H, Sévigny J. The ecto-nucleoside triphosphate diphosphohydrolase NTPDase2/ CD39L1 is expressed in a novel functional compartment within the liver. Hepatology. 2002;36(5):1135-1144.

19. Chang K, Pastan I. Molecular cloning of mesothelin, a differentiation antigen present on mesothelium, mesotheliomas, and ovarian cancers. Proc Natl Acad Sci U S A. 1996;93(1):136-140.

20. Pastan I, Hassan R. Discovery of mesothelin and exploiting it as a target for immunotherapy. Cancer Res. 2014;74(11):2907-2912.

21. Kaneko O, et al. A binding domain on mesothelin for CA125/MUC16.J Biol Chem. 2009;284(6):3739-3749.

22. Shimizu A, et al. Coexpression of MUC16 and mesothelin is related to the invasion process in pancreatic ductal adenocarcinoma. Cancer Sci. 2012;103(4):739-746.

23. Gubbels JA, et al. Mesothelin-MUC16 binding is a high affinity, $\mathrm{N}$-glycan dependent interaction that facilitates peritoneal metastasis of ovarian tumors. Mol Cancer. 2006;5(1):50.

24. Scholler N, Urban N. CA125 in ovarian cancer. Biomark Med. 2007;1(4):513-523.

25. Baghdasaryan A, et al. Curcumin improves sclerosing cholangitis in $\mathrm{Mdr2}^{-/}$mice by inhibition of cholangiocyte inflammatory response and portal myofibroblast proliferation. Gut. 2010;59(4):521-530.

26. Hassan R, Bera T, Pastan I. Mesothelin: a new target for immunotherapy. Clin Cancer Res. 2004;10(12 pt 1):3937-3942.

27. Hassan R, et al. Phase I study of SS1P, a recombinant anti-mesothelin immunotoxin given as a bolus I.V. infusion to patients with mesothelin-expressing mesothelioma, ovarian, and pancreatic cancers. Clin Cancer Res. 2007;13(17):5144-5149.

28. Kreitman RJ, Hassan R, Fitzgerald DJ, Pastan I. Phase I trial of continuous infusion anti-mesothelin recombinant immunotoxin SS1P. Clin Cancer Res. 2009;15(16):5274-5279.

29. Zhang J, et al. Megakaryocytic potentiating factor and mature mesothelin stimulate the growth of a lung cancer cell line in the peritoneal cavity of mice. PLoS One. 2014;9(8):e104388.

30. Akira S, Uematsu S, Takeuchi O. Pathogen recognition and innate immunity. Cell. 2006;124(4):783-801.

31. Bera TK, Pastan I. Mesothelin is not required for normal mouse development or reproduction. Mol Cell Biol. 2000;20(8):2902-2906.

32. Lujambio A, et al. Non-cell-autonomous tumor suppression by p53. Cell. 2013;153(2):449-460

33. McMullen MR, Pritchard MT, Wang Q, Millward CA, Croniger CM, Nagy LE. Early growth response-1 transcription factor is essential for ethanol-induced fatty liver injury in mice. Gastroenterology. 2005;128(7):2066-2076.

34. Lua I, et al. Characterization of hepatic stellate cells, portal fibroblasts, and mesothelial cells in normal and fibrotic livers. J Hepatol. 2016;64(5):1137-1146.

35. Peng $Z$, et al. Adenosine signaling contributes to ethanol-induced fatty liver in mice. JClin Invest. 2009;119(3):582-594.

36. Ramírez G, et al. Absence of Thy-1 results in TGF- $\beta$ induced MMP- 9 expression and confers a profibrotic phenotype to human lung fibroblasts. Lab Invest. 2011;91(8):1206-1218.

37. Shi Y, Massagué J. Mechanisms of TGF- $\beta$ signaling from cell membrane to the nucleus. Cell. 2003;113(6):685-700.

38. Rege TA, Hagood JS. Thy-1, a versatile modulator of signaling affecting cellular adhesion, proliferation, survival, and cytokine/ growth factor responses. Biochim Biophys Acta. 2006;1763(10):991-999.

39. Sanders YY, Tollefsbol TO, Varisco BM, Hagood JS. Epigenetic regulation of thy- 1 by histone deacetylase inhibitor in rat lung fibroblasts. $A m J$ Respir Cell Mol Biol. 2011;45(1):16-23.

40. Sanders YY, Kumbla P, Hagood JS. Enhanced myofibroblastic differentiation and survival in Thy-1(-) lung fibroblasts. Am J Respir Cell Mol Biol. 2007;36(2):226-235.

41. Yan X, Chen YG. Smad7: not only a regulator, but also a cross-talk mediator of TGF- $\beta$ signalling. Biochem J. 2011;434(1):1-10.

42. Zhou Y, Hagood JS, Lu B, Merryman WD, Murphy-Ullrich JE. Thy-1-integrin alphav beta5 interactions inhibit lung fibroblast contractioninduced latent transforming growth factor- $\beta 1$ activation and myofibroblast differentiation. J Biol Chem. 2010;285(29):22382-22393.

43. Dezso K, et al. Thy-1 is expressed in hepatic myofibroblasts and not oval cells in stem cell-mediated liver regeneration. Am J Pathol. 2007;171(5):1529-1537.

44. Dooley S, et al. Smad7 prevents activation of hepatic stellate cells and liver fibrosis in rats. Gastroenterology. 2003;125(1):178-191.

45. Er EE, Mendoza MC, Mackey AM, Rameh LE, Blenis J. AKT facilitates EGFR trafficking and degradation by phosphorylating and activating PIKfyve. Sci Signal. 2013;6(279):ra45.

46. Bharadwaj U, Marin-Muller C, Li M, Chen C, Yao Q. Mesothelin confers pancreatic cancer cell resistance to TNF- $\alpha$-induced apoptosis through Akt/PI3K/NF-kB activation and IL-6/Mcl-1 overexpression. Mol Cancer. 2011;10:106.

47. Chang MC, et al. Mesothelin enhances invasion of ovarian cancer by inducing MMP-7 through MAPK/ERK and JNK pathways. Biochem J. 2012;442(2):293-302.

48. Wang Y, Wang L, Li D, Wang HB, Chen QF. Mesothelin promotes invasion and metastasis in breast cancer cells. JInt Med Res. 2012;40(6):2109-2116.

49. Rao TD, et al. Expression of the carboxyterminal portion of MUC16/CA125 induces transformation and tumor invasion. PLoS One 2015;10(5):e0126633.

50. Nosten-Bertrand $M$, et al. Normal spatial learning despite regional inhibition of LTP in mice lacking Thy-1. Nature. 1996;379(6568):826-829.

51. Bast RC, et al. A radioimmunoassay using a monoclonal antibody to monitor the course of epithelial ovarian cancer. $N$ EnglJMed. 1983;309(15):883-887.

52. Molina R, et al. Cancer antigen 125 in serum and ascitic fluid of patients with liver diseases. Clin Chem. 1991;37(8):1379-1383.

53. Rinkevich Y, Mori T, Sahoo D, Xu PX, Bermingham JR, Weissman IL. Identification and prospective isolation of a mesothelial 


\section{RESEARCH ARTICLE}

precursor lineage giving rise to smooth muscle cells and fibroblasts for mammalian internal organs, and their vasculature. Nat Cell Biol. 2012;14(12):1251-1260.

54. Moore-Morris T, et al. Resident fibroblast lineages mediate pressure overload-induced cardiac fibrosis. J Clin Invest. 2014;124(7):2921-2934.

55. Li Y, Wang J, Asahina K. Mesothelial cells give rise to hepatic stellate cells and myofibroblasts via mesothelial-mesenchymal transition in liver injury. Proc Natl Acad Sci U S A. 2013;110(6):2324-2329.

56. Ali SR, et al. Developmental heterogeneity of cardiac fibroblasts does not predict pathological proliferation and activation. Circ Res. 2014;115(7):625-635.
57. Liu D, et al. Identification and characterization of mesenchymal-epithelial progenitor-like cells in normal and injured rat liver. Am J Pathol. 2015;185(1):110-128.

58. Zhou B, et al. Epicardial progenitors contribute to the cardiomyocyte lineage in the developing heart. Nature. 2008;454(7200):109-113.

59. Chan CK, et al. Endochondral ossification is required for haematopoietic stem-cell niche formation. Nature. 2009;457(7228):490-494.

60. Worthley DL, et al. Gremlin 1 identifies a skeletal stem cell with bone, cartilage, and reticular stromal potential. Cell. 2015;160(1-2):269-284.

61. Singh A, Lester C, Drapp R, Hu DZ, Glimcher LH, Jones D. Tetraspanin CD9 and ectonucleotidase CD73 identify an osteochondroprogenitor
The Journal of Clinical Investigation

population with elevated osteogenic properties. Development. 2015;142(3):438-443.

62. Yata Y, et al. DNase I-hypersensitive sites enhance alpha1(I) collagen gene expression in hepatic stellate cells. Hepatology. 2003;37(2):267-276.

63. Hatano E, Brenner DA. Akt protects mouse hepatocytes from TNF-alpha- and Fas-mediated apoptosis through NK-kB activation. Am J Physiol Gastrointest Liver Physiol. 2001;281(6):G1357-G1368.

64. Kim DW, Jeong S, Kim DS, Kim HS, Seo SB, Hahn $Y$. Inactivation of the MSLNL gene encoding mesothelin-like protein during African great ape evolution. Gene. 2012;496(1):17-21.

65. Pastan I, Zhang Y. Modulating mesothelin shedding to improve therapy. Oncotarget. 2012;3(2):114-115. 